\title{
A NEW CLASS OF SELF-ADJOINT BOUNDARY VALUE PROBLEMS
}

\author{
BY \\ WILLIAM T. REID
}

1. Introduction. Bliss $[1]\left({ }^{1}\right)$ has given a general definition of self-adjointness for a differential system of the form

$$
\begin{aligned}
y_{i}^{\prime} & =\sum_{j=1}^{n}\left[A_{i j}(x)+\lambda B_{i j}(x)\right] y_{j}, \\
s_{i}[y] & \equiv \sum_{j=1}^{n}\left[M_{i j} y_{j}(a)+N_{i j} y_{j}(b)\right]=0, \quad i=1, \cdots, n .
\end{aligned}
$$

In the paper above referred to, he has also discussed in detail a special class of self-adjoint problems termed definitely self-adjoint. In a subsequent paper [2], Bliss has given a modification of the definition of definite self-adjointness which is weaker than that previously considered, and has shown that most of the properties deduced in [1] are still valid for systems which are definitely self-adjoint according to the new definition.

If (1.1) is self-adjoint under the nonsingular real transformation $z_{i}=T_{i j}(x) y_{j}\left({ }^{2}\right)$, in both the original and modified definition of definitely self-adjoint problems Bliss has imposed the definiteness property of the system specifically on the matrix $S(x) \equiv\left\|S_{i j}(x)\right\| \equiv\left\|T_{k i}(x) B_{k j}(x)\right\|$. Now if $y_{i}(x)$, $(i=1, \cdots, n)$, is a solution of the differential equations of (1.1) for a value $\lambda$ it follows immediately that

$$
\int_{a}^{b} y_{i} T_{j i}\left[y_{j}^{\prime}-A_{j k} y_{k}\right] d x=\lambda \int_{a}^{b} y_{i} S_{i j} y_{j} d x .
$$

It may be readily verified that the definiteness property of $S(x)$ assumed by Bliss could equally well have been phrased as a definiteness property of the quadratic functional

$$
\int_{a}^{b} y_{i} S_{i j} y_{j} d x
$$

If $H[y]$ denotes the first member of (1.2), the theory of pencils of quad-

Presented to the Society, September 12, 1940; received by the editors May 14, 1941; corrections received June 17, 1942.

${ }^{1}$ Numbers in square brackets refer to the bibliography at the end of this paper.

${ }^{2}$ In the introduction, and throughout the paper where matrix notation is not more convenient, the repetition of a subscript in a single term of an expression denotes summation with respect to that subscript over its range of definition. 
ratic forms in a finite number of variables suggests that a differential system (1.1) for which $H[y]$ satisfies suitable conditions of definiteness may possess properties analogous to those enjoyed by the class of definitely self-adjoint problems as defined by Bliss [2]. The prime aim of this paper is to show that such is indeed the case. The class of self-adjoint problems herein studied, for which the definiteness property is placed on the functional $H[y]$, is termed $H$-definitely self-adjoint. Moreover, it is to be emphasized that the study of $H$-definitely self-adjoint problems affords new results for systems which are definitely self-adjoint in the sense of Bliss.

The definition of an $H$-definitely self-adjoint system is given in $\$ 2$, and properties of the functional $H[y]$ are presented in $\$ 3$. Preliminary results for such a system are obtained in $\$ 4$; one of the most important results therein contained is that of Theorem 4.3 , which states that for an $H$-definitely selfadjoint system the matrix $B(x)$ must be such that its square is identically zero on the interval $a b$. This result, which at first seems startling in aspect, admits certain important consequences for definitely self-adjoint systems. The fundamental properties of an $H$-definitely self-adjoint system, such as the reality of the characteristic values, the equality of the index and multiplicity of a characteristic value, and a type of completeness property of the totality of characteristic solutions for such a system, are contained in $\$ 5$; $\S 6$ is devoted to the discussion of the existence of characteristic values for such a system. Results for definitely self-adjoint systems are given in $\S 7$, whereas $\S 8$ is concerned with a special definitely self-adjoint problem which is related to a given system (1.1), although the system (1.1) itself may be neither definitely nor $H$-definitely self-adjoint. By the use of the results of the preceding section, extremizing properties of the characteristic values and characteristic solutions of an $H$-definitely self-adjoint system are established in $\S 9$. In $\S 10$ it is shown that an important instance of the type of boundary value problems associated with the calculus of variations previously studied by the author [9] is $H$-definitely self-adjoint. The connection between the class of problems herein treated and the boundary problems associated with a single linear differential equation of even order which have been studied by Krein [7] and Kamke [6] is indicated briefly in $\$ 11$. Finally, $\$ 12$ is devoted to the extension of the notion of $H$-definite self-adjointness to the case of systems whose coefficients are complex-valued.

For simplicity, matrix notation is used almost exclusively in this paper. Square matrices with $n$ rows and columns are denoted by capital italic letters, and the element in the $i$ th row and $j$ th column is denoted by the letter representing the matrix with the subscript $i j$. Lower case italic letters signify vectors with $n$ components, the $i$ th component being signified by a subscript $i$. If $M \equiv\left\|M_{i j}\right\|, u \equiv\left[u_{i}\right]$, the vectors $\left[M_{i j} u_{j}\right]$ and $\left[u_{j} M_{j i}\right]$ are denoted by $M u$ and $u M$, respectively. The scalar product $u_{j} v_{j}$ of two vectors is written $u v$. If $\alpha$ is a scalar, $\bar{\alpha}$ is its complex conjugate, and for a vector $u$ we write $\bar{u}$ 
for $\left[\bar{u}_{i}\right]$. For a matrix $M=\left\|M_{i j}\right\|$ we use $\tilde{M}$ for the transpose matrix $\left\|M_{j i}\right\|$. Finally, if the elements of $M$ are differentiable functions, the matrix of derivatives is denoted by $M^{\prime}$; similarly, if the components of $u$ are differentiable functions, we write $u^{\prime} \equiv\left[u_{i}^{\prime}\right]$. The norm of a vector $u,[u \bar{u}]^{1 / 2}$, is written norm $\{u\}$.

2. Definition of $H$-definitely self-adjoint systems. In the following pages it will be assumed that the elements of the matrices $A(x)$ and $B(x)$ are real single-valued continuous functions on the finite interval $a \leqq x \leqq b$ and that the elements of $B(x)$ are not all identically zero on this interval. The elements of the matrices $M$ and $N$ are supposed to be real constants such that the $n \times 2 n$ matrix $\left\|M_{i j} N_{i j}\right\|$ is of rank $n$. Moreover, because of its frequent occurrence, we write $\mathcal{L}[y]$ for the vector differential operator $y^{\prime}-A(x) y$. The boundary value problem to be considered in this paper may then be written

$$
\mathcal{L}[y]=\lambda B(x) y, \quad s[y] \equiv M y(a)+N y(b)=0 .
$$

The system adjoint to (2.1) is

$$
\mathcal{X}[z]=-\lambda z B(x), \quad t[z] \equiv z(a) P+z(b) Q=0,
$$

where $\mathcal{X}[z]$ is the adjoint differential operator $z^{\prime}+z A(x)$, and $p \equiv\left(p_{i}\right) \equiv\left(P_{i j}\right)$, $q \equiv\left(q_{i}\right) \equiv\left(Q_{i j}\right),(j=1, \cdots, n)$, are $n$ linearly independent solutions of the algebraic equations $M p-N q=0$.

According to the modified definition of Bliss [2] the system (2.1) is definitely self-adjoint with a matrix $T$, or simply definitely self-adjoint, whenever the following conditions are satisfied:

(i) The system is self-adjoint under the nonsingular real transformation $z=T(x) y$; that is, for arbitrary values of $\lambda$ a vector $y$ satisfies the differential equations (or boundary conditions) of (2.1) if and only if the associated vector $z=T y$ satisfies the differential equations (or boundary conditions) of (2.2). The elements of $T(x)$ are supposed to be of class $C^{1}$ on the interval $a b$.

(ii) The matrix $S(x)=\tilde{T}(x) B(x)$ is symmetric on $a b$.

(iii) The quadratic form $u S(x) u$ is positive semi-definite on $a b$.

(iv) There exists no nonidentically vanishing solution $y$ of $\mathcal{L}[y]=0$, $s[y]=0$ such that $B(x) y(x) \equiv 0$ on $a b$.

The wording of hypothesis (iv) differs slightly from that used by Bliss [2, property (3), p. 414]. However, when (ii) and (iii) are satisfied the above hypothesis (iv) is readily seen to be equivalent to the property (3) of Bliss. For the present treatment the form (iv) is preferable.

It is to be remarked $[1$, p. 569$]$ that a nonsingular matrix $T(x)$ whose elements are of class $C^{1}$ on $a b$ satisfies condition (i) if and only if

$$
\begin{gathered}
T A+\tilde{A} T+T^{\prime} \equiv 0, \quad T B+\tilde{B} T \equiv 0 \quad \text { on } \quad a b, \\
M T^{-1}(a) \tilde{M}=N T^{-1}(b) \tilde{N} .
\end{gathered}
$$


Consequently, whenever (ii) is also satisfied by $T$ we have

$$
S \equiv \tilde{T} B \equiv \widetilde{B} T \equiv-T B .
$$

For $y$ a solution of (2.1) corresponding to a characteristic value $\lambda$, relation (1.2) becomes in matrix and vector notation

$$
\int_{a}^{b} y \tilde{T} \mathcal{L}[y] d x=\lambda \int_{a}^{b} y S y d x
$$

Now the above hypothesis (iii) clearly implies a positive semi-definite character of the quadratic functional

$$
\int_{a}^{b} y S y d x
$$

Indeed, (ii) and (iii) together imply that this functional is positive for all vectors $y$ whose components are continuous on $a b$ and such that $B(x) y(x) \not \equiv 0$ on this interval.

The quadratic functional upon which certain assumptions of definiteness are to be imposed in this paper is

$$
H[y] \equiv \int_{a}^{b} y \tilde{T} \mathcal{L}[y] d x,
$$

which appears as the left-hand member of (2.5).

For convenience, we shall denote by $L$ the linear vector space consisting of all vectors $y$ satisfying the following conditions: $(\alpha)$ the components of $y$ are real-valued and of class $C^{1}$ on $a b ;(\beta) s[y]=0 ;(\gamma)$ there exists a corresponding vector $g(x)$ with real-valued continuous components such that $\mathcal{L}[y]=B g$ on $a b$.

Instead of the above hypothesis (iii) we shall now assume the following condition:

(iii)' The quadratic functional $H[y]$ is positive for arbitrary vectors $y$ of $L$ such that $B(x) y(x) \not \equiv 0$ on $a b$.

A system (2.1) which satisfies hypotheses (i), (ii), (iii)' and (iv) will be termed $H$-definitely self-adjoint with the matrix $T$, or simply $H$-definitely selfadjoint; the prefix " $H$-" indicates that it is the functional $H[y]$ which possesses the definiteness property. Correspondingly, a system which is definitely self-adjoint as defined by Bliss [2] might be termed $S$-definitely self-adjoint. It is to be pointed out that in the treatment of definitely self-adjoint systems, as well as in the present discussion of $H$-definitely self-adjoint systems, the space $L$ occupies a central position.

Now a linear change of parameter in (2.1), replacing $\lambda$ by $\lambda+\lambda_{0}$, has the effect of substituting $\mathcal{L}[y]-\lambda_{0} B y$ for $\mathcal{L}[y]$. Hence it is to be emphasized that as far as the qualitative properties of (2.1) are concerned the hypothesis (iii)' 
is no stronger than the assumption that there is some value of $\lambda_{0}$ such that the functional

$$
H\left[y: \lambda_{0}\right] \equiv \int_{a}^{b} y \tilde{T}\left(\mathcal{L}[y]-\lambda_{0} B y\right) d x
$$

satisfies the definiteness property of (iii)'.

In view of equations (2.3), a system (2.1) which is self-adjoint with a matrix $T$ is also self-adjoint with the matrix $-T, \tilde{T}$ or $-\tilde{T}$. In particular, if (2.1) is $H$-definitely self-adjoint with a matrix $T$ it is also $H$-definitely selfadjoint with the matrix $-\tilde{T}$. If hypotheses (i), (ii) and (iv) are satisfied by (2.1) with a matrix $T$ and the functional (2.6) is negative for arbitrary vectors $y$ of $L$ such that $B y \neq \equiv 0$, this functional can be replaced by one for which (iii)' as stated is satisfied by using the transformation matrix $-T$ instead of $T$. Moreover, if (2.1) is $H$-definitely self-adjoint with a matrix $T$ then the adjoint system (2.2), written in the form (2.1), is $H$-definitely self-adjoint with the matrix $\tilde{T}^{-1}$. It may be readily verified that analogous results hold for definitely self-adjoint systems.

We shall denote by $L^{2}$ the linear subspace of $L$ consisting of all vectors $y$ with real components and satisfying a system $\mathcal{L}[y]=B g, s[y]=0$, where $g(x)$ is also a vector of the space $L$. Clearly each real characteristic solution of (2.1) belongs to $L^{2}$ as well as to $L$. In the subsequent discussion the space $L^{2}$ first occurs in Theorem 5.4 .

3. Properties of the quadratic functional $H[y]$. If $u$ and $v$ are vectors whose components are of class $C^{1}$ on $a b$, let $H[u ; v]$ denote the bilinear expression

$$
H[u ; v]=\int_{a}^{b} u \tilde{T} \mathcal{L}[v] d x .
$$

In general $H[u ; v] \neq H[v ; u]$. However, we do have the following property.

LеммA 3.1. For a system (2.1) satisfying conditions (i) and (ii) the bilinear functional $H[u ; v]$ is symmetric on the linear vector space $L$.

For suppose that $u$ and $v$ belong to $L$, and that $\mathcal{L}[u]=B g, \mathcal{L}[v]=B h$. Then $w=T v$ satisfies the system $\mathcal{X}[w]=-h \tilde{T} B, t[w]=0$. By a familiar argument it then follows (see Bliss [1, p. 567]) that

$$
\int_{a}^{b} w B g d x-\int_{a}^{b} h S u d x=\left.w u\right|_{x=a} ^{x=b}=0 .
$$

The result of the lemma is then immediate since

$$
\begin{aligned}
& \int_{a}^{b} h S u d x=\int_{a}^{b} u \tilde{T} B h d x=H[u ; v], \\
& \int_{a}^{b} w B g d x=\int_{a}^{b} v \tilde{T} B g d x=H[v ; u] .
\end{aligned}
$$


LEMMA 3.2. Hypotheses (ii) and (iii)' imply $H[y] \geqq 0$ on $L$.

For consider an arbitrary vector $y$ of $L$. If $B y \neq 0$ on $a b$, then (iii)' implies $H[y]>0$. On the other hand, if $B y \equiv 0$ on this interval the symmetry of $S$ insures

$$
H[y] \equiv \int_{a}^{b} y \tilde{T} B g d x=\int_{a}^{b} g \tilde{T} B y d x=0 .
$$

The following result is an immediate consequence of Lemma 3.1 and the linearity of $L$.

Lемма 3.3. If the system (2.1) satisfies (i), (ii), and $H[y] \geqq 0$ on $L$, then

$$
\{H[u ; v]\}^{2} \leqq H[u] H[v]
$$

for arbitrary vectors $u$ and $v$ of $L$.

Lemma 3.4. If (2.1) satisfies hypotheses (i) and (ii) and $y, y^{*}$ are characteristic functions corresponding to distinct characteristic values $\lambda, \lambda^{*}$, then

$$
\int_{a}^{b} y^{*} S y d x=0, \quad H\left[y^{*} ; y\right]=0 .
$$

The first equality of (3.2) follows by Theorem 8 of Bliss [1], and the second relation is then immediate since $y^{*} \tilde{T} \mathcal{L}[y]=\lambda y^{*} S y$. It is to be remarked that this result is true quite independent of the reality of the characteristic values and characteristic functions involved.

4. Preliminary results. In this section we shall present some results for $H$-definitely self-adjoint systems which, although of individual significance, are preliminary to the rest of the paper.

TheOREM 4.1. If (2.1) is $H$-definitely self-adjoint, then $\lambda=0$ is not a characteristic value of this system.

For suppose $\lambda=0$ were a characteristic value for such a system, and denote by $y$ a corresponding real characteristic solution. The condition (iv) implies $B y \neq 0$ on $a b$, and as $y$ clearly belongs to $L$ it follows by (iii)' that $H[y]>0$ : On the other hand, $H[y]=0$ since $\mathcal{L}[y] \equiv 0$. Hence $\lambda=0$ is not a characteristic value.

In connection with this theorem, we also have the following result.

THEOREM 4.2. If (2.1) satisfies conditions (i) and (ii), $H[y] \geqq 0$ on $L$, and $\lambda=0$ is not a characteristic value, then this system is $H$-definitely self-adjoint.

If $\lambda=0$ is not a characteristic value for (2.1), then clearly condition (iv) is satisfied. Moreover, suppose that $y$ is a particular vector of $L$ such that $H[y]=0$. Then by Lemma 3.3 it follows that $H[y ; v]=0$ for arbitrary vectors $v$ of $L$. But for an arbitrary vector $g$ whose components are continuous there 
exists, if $\lambda=0$ is not a characteristic value, a unique solution of $\mathcal{L}[v]=B g$, $s[v]=0$; for this vector $v, H[y ; v]=\int_{a}^{b} y S g d x$. Hence $0 \equiv y S \equiv \tilde{T} B y$, and therefore $B y \equiv 0$ on $a b$. That is, if $y$ is a vector of $L$ for which $H[y]=0$ it must be true that $B y \equiv 0$. Hence condition (iii)' is also satisfied by such a system, and it is $H$-definitely self-adjoint.

As a consequence of Theorem 4.1 , for an $H$-definitely self-adjoint system the functional $H[y]$ is afforded an alternate representation. Let $G(x, t) \equiv\left\|G_{i j}(x, t)\right\|$ denote the Green's matrix (see, for example, Bliss [1, pp. 577-581]) for the incompatible system

$$
\mathcal{L}[y]=0, \quad s[y]=0 .
$$

Then a vector $y$ belongs to $L$ if and only if it is of the form

$$
y(x)=\int_{a}^{b} K(x, t) g(t) d t
$$

where $K(x, t)=G(x, t) B(t)$, and the components of $g$ are continuous on $a b$. We may then write

$$
\begin{aligned}
H[y] & =\int_{a}^{b} y \tilde{T} B g d x=\int_{a}^{b} g S y d x \\
& =\int_{a}^{b} \int_{a}^{b} g(x) S(x) K(x, t) g(t) d x d t \\
& =\int_{a}^{b} \int_{a}^{b} g(x) K_{1}(x, t) g(t) d x d t,
\end{aligned}
$$

where $K_{1}(x, t)=S(x) K(x, t)=S(x) G(x, t) B(t)$. Similarly, if $u$ and $v$ are vectors belonging to $L$ and $\mathcal{L}[u]=B g, \mathcal{L}[v]=B h$, we have

$$
H[u ; v]=\int_{a}^{b} \int_{a}^{b} h(x) K_{1}(x, t) g(t) d x d t .
$$

Now corresponding to arbitrary vectors $g, h$ whose components are continuous there exist unique corresponding vectors $u, v$ of $L$ satisfying the above conditions. Since by Lemma 3.1 we have $H[u ; v]=H[v ; u]$ it then follows that

$$
K_{1}(x, t) \equiv \tilde{K}_{1}(t, x),
$$

that is,

$$
S(x) K(x, t) \equiv \tilde{K}(t, x) S(t) .
$$

Indeed, in the proof of (4.4) we have used in addition to hypotheses (i), (ii), only the condition that $\lambda=0$ is not a characteristic value of (2.1). Relation (4.4) has been obtained by Bliss [1, p. 580], and it may be readily verified that his proof also uses only these conditions on the system (2.1). 
For convenience in the presentation of the following two lemmas the functional (4.3) will be denoted by $J[g]$; similarly, the quantity $\left(4.3^{\prime}\right)$ will be written $J[h ; g]$. The following result will be stated without proof, since it follows readily from well known properties of Lebesgue integrals.

LEMma 4.1. If $J[g] \geqq 0$ for all vectors $g$ whose components are continuous on $a b$, then this integral, taken in the sense of Lebesgue, is non-negative for all vectors $g$ whose components are of integrable square on this interval.

Now denote by $L^{\prime}$ the extension of $L$ obtained by considering the totality of vectors $y$ such that: $\left(\alpha^{\prime}\right)$ the components of $y$ are real-valued and absolutely continuous on $a b ;\left(\beta^{\prime}\right) s[y]=0 ;\left(\gamma^{\prime}\right)$ there exists a corresponding vector $g$ whose components are real-valued, of Lebesgue integrable square on $a b$, such that $\mathcal{L}[y]=B g$ almost everywhere on this interval. In view of Lemma 4.1, and the fact that we still have $y(x)=\int_{a}^{b} K(x, t) g(t) d t$ for a vector $y$ of $L^{\prime}$, it follows that the results of Lemmas 3.1, 3.2 and 3.3 remain valid for $y, u$ and $v$ vectors in $L^{\prime}$. Moreover, since $\lambda=0$ is not a characteristic value for an $H$-definitely self-adjoint problem, it follows as in the proof of Theorem 4.2 that $H[y]=0$ for a vector $y$ of $L^{\prime}$ if and only if $B y \equiv 0$ on $a b$. That is, as far as the results previously established are concerned, in the definition of $H$-definitely self-adjoint systems one might without further restriction have used the vector space $L^{\prime}$ instead of $L$. As a matter of fact, this remark is valid for all the results obtained in the present paper. Specifically, in this connection, it is to be pointed out that the results of Bliss used in $\$ 7$ are valid for the space $L^{\prime}$ instead of $L$.

Because of the special form of $K_{1}(x, t)$, and the fact that if the components of $b(x)$ are integrable on $a b$ then $y(x)=\int_{a}^{b} G(x, t) b(t) d t$ is a vector whose components are absolutely continuous, satisfies $\mathcal{L}[y]=b(x)$ almost everywhere on $a b$, and $s[y]=0$, all the preceding results may be proved for a much more general linear vector space than $L^{\prime}$. In particular, they all hold for the space of vectors $y$ satisfying the above conditions $\left(\alpha^{\prime}\right),\left(\beta^{\prime}\right)$, and the condition obtained by replacing in $\left(\gamma^{\prime}\right)$ the phrase "of Lebesgue integrable square" by "Lebesgue integrable." However, for a number of the subsequent results to remain valid, it is necessary to restrict the involved vectors to the space $L^{\prime}$.

We shall denote by $K_{1 i j}(x, x+)$ the limiting values of $K_{1 i j}(x, t)$ as $t$ tends to $x$ through values greater than $x$, and write $K_{1}(x, x+) \equiv\left\|K_{1 i j}(x, x+)\right\|$. The quantities $K_{1 i j}(x, x-)$ and $K_{1}(x, x-)$ are defined in a corresponding fashion. Since $K_{1}(x, t)=S(x) G(x, t) B(t)$ it follows that the elements of $K_{1}$ have discontinuities at most along the line $x=t$. Moreover, if $K_{1}(x, t)$ is taken to be equal to $K_{1}(x, x+)$ along $x=t$, then the elements of the resulting matrix are continuous in $(x, t)$ on the region $R_{1}: x \leqq t \leqq b, a \leqq x \leqq b$. Similarly, if $K_{1}(x, t)$ is taken to be equal to $K_{1}(x, x-)$ along $x=t$, then the elements of the resulting matrix are continuous in $(x, t)$ on $R_{2}: a \leqq t \leqq x ; a \leqq x \leqq b$.

LEмma 4.2. If the functional $J[g]$ defined by (4.3) be positive semi-definite for 
arbitrary vectors $g$ whose components are continuous on $a b$, then $K_{1}(x, x-)$ $=K_{1}(x, x+)$ on $a \leqq x \leqq b$; that is, if $K_{1}(x, t)$ be taken as equal to this common value along the line $x=t$, then the elements of $K_{1}$ are continuous in $(x, t)$ on $a \leqq x, t \leqq b$. Moreover, the matrix $K_{1}(x, x)$ thus defined is symmetric and positive semi-definite on $a b$.

For convenience, we write $J[g]=J_{1}[g]+J_{2}[g]$, where $J_{1}$ and $J_{2}$ are the integrals of the integrand of (4.3) taken over the above defined regions $R_{1}$ and $R_{2}$, respectively. The integrals $J_{1}[h ; g], J_{2}[h ; g]$ are defined similarly. Now consider a point $\left(x_{0}, x_{0}\right)$ with $a<x_{0}<b$. For an arbitrary constant vector $g_{0}$, denote by $g_{k}(x),(k=1,2, \cdots)$, the vector whose components are identically zero except on $\left|x-x_{0}\right| \leqq d_{k}$, where $d_{k}=c / k$ and $c$ is the smaller of the numbers $x_{0}-a, b-x_{0}$, while on $x_{0}-d_{k} \leqq x<x_{0}$ we define $g_{k}=\left(1 / d_{k}\right) g_{0}$, and on $x_{0} \leqq x \leqq x_{0}+d_{k}$ we set $g_{k}=\left(-1 / d_{k}\right) g_{0}$. Because of the continuity properties of the elements of $K_{1}$ as described above, it is readily calculated that $\lim _{k \rightarrow \infty} J_{1}\left[g_{k}\right]=0=\lim _{k \rightarrow \infty} J_{2}\left[g_{k}\right]$, and consequently, $\lim _{k \rightarrow \infty} J\left[g_{k}\right]=0$. Now for a second arbitrary constant vector $h_{0}$, define $h_{k}(x) \equiv 0$ except on $x_{0}-d_{k} \leqq x \leqq x_{0}+d_{k}, h_{k}(x) \equiv\left(1 / d_{k}\right) h_{0}$ on this subinterval. Clearly there exists a constant $\kappa$ such that $H\left[h_{k}\right] \leqq \kappa,(k=1,2, \cdots)$. Moreover, in view of the positive semi-definite character of $J$ proved in Lemma 4.1 , we then have $\left\{J\left[h_{k} ; g_{k}\right]\right\}^{2} \leqq J\left[h_{k}\right] J\left[g_{k}\right] \leqq \kappa J\left[g_{k}\right],(k=1,2, \cdots)$, and hence $\lim _{k \rightarrow \infty} J\left[h_{k} ; g_{k}\right]$ $=0$. Again, using the continuity properties of the elements of $K_{1}$ described above, it is found that $\lim _{k \rightarrow \infty} J_{1}\left[h_{k} ; g_{k}\right]=-h_{0} K_{1}\left(x_{0}, x_{0}+\right) g_{0}$, and $\lim _{k \rightarrow \infty} J_{2}\left[h_{k} ; g_{k}\right]=h_{0} K_{1}\left(x_{0}, x_{0}-\right) g_{0}$. Thus for arbitrary constant vectors $h_{0}$, $g_{0}$ we have $h_{0}\left[K_{1}\left(x_{0}, x_{0}-\right)-K_{1}\left(x_{0}, x_{0}+\right)\right] g_{0}=0$, and consequently $K_{1}(x, x-)$ $-K_{1}(x, x+) \equiv 0$ on $a<x<b$, whence it in turn follows that this relation is also true at the end values $a$ and $b$. In the following we shall write $K_{1}(x, x)$ for this common limiting value along the line $x=t$. Returning to the above defined sequence $\left\{h_{k}\right\}$, we see that for $a<x_{0}<b$ we have $4 h_{0} K_{1}\left(x_{0}, x_{0}\right) h_{0}=\lim _{k \rightarrow \infty} J\left[h_{k}\right]$ $\geqq 0$ for arbitrary vectors $h_{0}$. Hence, the matrix $K_{1}(x, x)$ is positive semidefinite on $a<x<b$, and by continuity this property holds on the closed interval $a b$. The symmetry of this matrix follows from (4.4).

Clearly the above result applies to any positive semi-definite kernel matrix $K_{1}(x, t)$ such that $K_{1}(x, t)=\widetilde{K}_{1}(t, x)$, and which possesses the continuity properties described immediately preceding the lemma.

THEOREM 4.3. For an H-definitely self-adjoint system (2.1) the matrix $B(x)$ must satisfy the condition $B B \equiv 0$ on $a b$; in particular, the rank of $B(x)$ at any point of this interval cannot be greater than $[n / 2]$, the largest integer not exceeding the value $n / 2$.

Since for an $H$-definitely self-adjoint system we have $K_{1}(x, t)$ $=S(x) G(x, t) B(t)$, and as $G(x, x-)-G(x, x+) \equiv I$ on $a b$ (see, for example, $\left[1\right.$, p. 578]), we have from Lemma 4.2 that $0 \equiv K_{1}(x, x-)-K_{1}(x, x+)$ $\equiv S(x) B(x) \equiv \tilde{T}(x) B(x) B(x)$ on $a b$. As $T$ is nonsingular, it then follows that 
$B B \equiv 0$ on this interval. Algebraically, it is readily seen that this condition implies that at each point of $a b$ the rank of $B$ cannot exceed [ $n / 2]$.

This result, which at first notice seems remarkable, first occurred to the author in considering the results which will be presented in $\$ 8$. Indeed, because of this relatively strong condition imposed upon $B(x)$ by the $H$-definite self-adjoint character of (2.1), one might conclude that this class of boundary value problems is too restrictive to be of great significance. That this is not so, however, is borne out by the fact that this class of problems includes those of the type discussed in $\$ 10$. Moreover, the additional results obtained in $\$ 7$ concerning systems which are definitely self-adjoint in the sense of Bliss also show the significance of such problems.

5. Properties of $H$-definitely self-adjoint systems. We shall now proceed to establish some fundamental properties of systems (2.1) which are $H$-definitely self-adjoint.

THEOREM 5.1. All the characteristic values of an H-definitely self-adjoint system are real, and the corresponding characteristic functions may be chosen real.

Suppose $\lambda=\lambda_{1}+(-1)^{1 / 2} \lambda_{2},\left(\lambda_{2} \neq 0\right)$, is a characteristic value of (2.1), and $y=u+(-1)^{1 / 2} v$ is a corresponding characteristic solution. Then $\bar{y}=u-(-1)^{1 / 2} v$ is a characteristic solution of this system corresponding to the complex conjugate value $\bar{\lambda}$ of the characteristic parameter. As $\lambda \neq \bar{\lambda}$, it follows from Lemma 3.4 that $H[\bar{y} ; y]=0$. Since

$$
\mathcal{L}[u]=\lambda_{1} B u-\lambda_{2} B v, \quad \mathcal{L}[v]=\lambda_{2} B u+\lambda_{1} B v, \quad s[u]=0=s[v],
$$

the vectors $u, v$ belong to $L$. Consequently, in view of Lemma 3.1, $H[\bar{y} ; y]=H[u]+H[v]$. It then follows from condition (iii)' and Lemma 3.2 that $B u \equiv 0, B v \equiv 0$ on $a b$; that is, $u$ and $v$ are individually solutions of (2.1) for $\lambda=0$. It is then a consequence of Theorem 4.1 that $u \equiv 0, v \equiv 0$, which is a contradiction to the assumption that $y=u+(-1)^{1 / 2} v$ is a characteristic solution for the value $\lambda$. Hence all the characteristic values of an $H$-definitely self-adjoint system are real, and because of the reality of the coefficients of such a system the corresponding characteristic solutions may be chosen real.

In the future, when we speak of a characteristic solution of an $H$-definitely self-adjoint system, it will be understood that this solution is real.

THEOREM 5.2. If $\lambda$ is a characteristic value of an $H$-definitely self-adjoint system, and $y$ a corresponding characteristic solution, then $H[y]>0$ and $\int_{a}^{b} y S y d x$ has the sign of $\lambda$.

Since, by Theorem $4.1, \lambda=0$ is not a characteristic value, for a characteristic solution $y$ of $(2.1)$ we have $B y \neq 0$ on $a b$, and hence $H[y]>0$ by (iii)'. The rest of the theorem is an immediate consequence of (2.5).

Let $Y(x, \lambda)$ be a matrix whose columns are $n$ linearly independent solutions of the differential equations of (2.1), and whose elements are perma- 
nently convergent power series in $\lambda$. Such a matrix is determined, for example, by the initial condition $Y(a, \lambda)=I$. By definition, the multiplicity of a characteristic value of $(2.1)$ is equal to its multiplicity as a zero of the characteristic determinant $|M Y(a, \lambda)+N Y(b, \lambda)|$, which is a permanently convergent power series in $\lambda$. The index of $\lambda$ as a characteristic value of (2.1) is equal to the number of corresponding linearly independent solutions of this system.

THEOREM 5.3. For an H-definitely self-adjoint system (2.1) the index of a characteristic value is equal to its multiplicity.

The proof is the same as that of Theorem 10 in Bliss [1], down to the last equation on page 572. On the assumption that the result of the theorem is not true, this equation states that there exists a characteristic solution $y$ of (2.1) such that $\int_{a}^{b} y S y d x=0$. This, however, is impossible in view of the above Theorem 5.2.

THEOREM 5.4. If the components of $f$ are continuous on ab, and the condition

$$
\int_{a}^{b} f S y d x=0
$$

is satisfied by every characteristic solution $y$ of an $H$-definitely self-adjoint system, then this relation is also satisfied by every vector $y$ of $L^{2}$.

In view of the preceding theorem, the condition (5.1) for all characteristic solutions of (2.1) implies, as in the proof of Theorem 11 of Bliss [1], that the nonhomogeneous system

$$
\mathcal{L}[y]=\lambda B y+B f, \quad s[y]=0,
$$

has a solution $y(x, \lambda)$ of the form

$$
y(x, \lambda)=u_{0}(x)+\lambda u_{1}(x)+\cdots+\lambda^{\mu} u_{\mu}(x)+\cdots ;
$$

the components of $u_{\mu}(x),(\mu=0,1, \cdots)$, are of class $C^{1}$ on $a b$, and this series converges absolutely and uniformly on any region of the form $a \leqq x \leqq b$, $|\lambda| \leqq \rho$. Moreover, if we write $u_{-1}(x)=f(x)$ and $v_{\mu}(x)=T(x) u_{\mu}(x),(\mu=-1,0$, $1, \cdots)$, then

$$
\begin{aligned}
& \mathcal{L}\left[u_{\mu}\right]=B u_{\mu-1}, \quad s\left[u_{\mu}\right]=0, \quad \mu=0,1, \cdots, \\
& \mathscr{X}\left[v_{v}\right]=-v_{v-1} B, \quad t\left[v_{v}\right]=0, \quad \nu=0,1, \cdots .
\end{aligned}
$$

In view of the boundary conditions we also have

As

$$
\int_{a}^{b} u_{\nu-1} S u_{\mu} d x=\int_{a}^{b} u_{\nu} S u_{\mu-1} d x, \quad \mu, \nu=0,1, \cdots
$$

$$
\int_{a}^{b} u_{\nu-1} S u_{\mu} d x=\int_{a}^{b} u_{\nu-1} \tilde{T} B u_{\mu} d x=\int_{a}^{b} u_{\nu-1} \tilde{T} \mathcal{L}\left[u_{\mu+1}\right] d x
$$




$$
\int^{b} \cdot u_{\nu} S u_{\mu-1} d x=\int_{a}^{b} u_{\nu} \tilde{T} B u_{\mu-1} d x=\int_{a}^{b} u_{\nu} \tilde{T} \mathcal{L}\left[u_{\mu}\right] d x
$$

we have

$$
H\left[u_{\nu-1} ; u_{\mu+1}\right]=H\left[u_{\nu} ; u_{\mu}\right], \quad \mu, \nu=1,2, \cdots .
$$

By Lemma 3.1 it also follows that $H\left[u_{\nu} ; u_{\mu}\right]=H\left[u_{\mu} ; u_{\nu}\right]$. Now set

$$
W_{\mu}=H\left[u_{0} ; u_{\mu}\right], \quad \mu=0,1, \cdots .
$$

By the above relations we have

$$
W_{\mu+\nu}=H\left[u_{\nu} ; u_{\mu}\right], \quad \mu, \nu=0,1, \cdots,
$$

and it results from Lemma 3.3 that

$$
\left[W_{2 \mu}\right]^{2}=\left[W_{(\mu-1)+(\mu+1)}\right]^{2} \leqq W_{2 \mu-2} W_{2 \mu+2}, \quad \mu=1,2, \cdots .
$$

Writing the differential equations of (5.2) in integral form, and employing the uniform convergence of (5.3) in a region of the form $a \leqq x \leqq b,|\lambda| \leqq \rho$, it follows that $\mathcal{L}[y]$ is a permanently convergent power series in $\lambda$ given by

$$
\begin{array}{r}
\mathcal{L}\left[u_{0}\right]+\lambda \mathcal{L}\left[u_{1}\right]+\cdots+\lambda^{\mu} \mathcal{L}\left[u_{\mu}\right]+\cdots \\
=B f+\lambda B u_{0}+\cdots+\lambda^{\mu} B u_{\mu-1}+\cdots .
\end{array}
$$

From its specific form, it is seen that the series (5.7) has convergence properties of the sort indicated above for the series (5.3). Consequently, the series

$$
W_{0}+\lambda W_{1}+\lambda^{2} W_{2}+\cdots, \quad W_{0}+\lambda^{2} W_{2}+\cdots,
$$

the first of which is equal to $H\left[u_{0} ; y\right]$, are permanently convergent power series in $\lambda$.

If $W_{2} \neq 0$ it follows from (5.6) that $W_{2 \mu} \neq 0,(\mu=1,2, \cdots)$, and the second series of (5.8) is seen to diverge for $\lambda=\left(W_{2} / W_{4}\right)^{1 / 2}$. Hence the permanent convergence of this series is possible only if $0=W_{2}=H\left[u_{1}\right]$. Condition (iii)' then implies that $B u_{1} \equiv 0$ on $a b$. Moreover, by Lemma 3.3 we have $H\left[y ; u_{1}\right]=0$ for arbitrary vectors $y$ of $L$. As $\mathcal{L}\left[u_{1}\right]=B u_{0}$, we may also state this condition as

$$
0=\int_{a}^{b} y \tilde{T} \mathcal{L}\left[u_{1}\right] d x=\int_{a}^{b} y S u_{0} d x
$$

for arbitrary vectors $y$ of $L$. In particular, for $y=u_{0}$ we have

$$
\int_{a}^{b} u_{0} S u_{0} d x=0
$$

Now suppose $y$ is any vector belonging to the space $L^{2}$, and $g(x)$ is a vector of $L$ such that $\mathcal{L}[y]=B g$. By Lemma 3.1 we then have 


$$
\begin{aligned}
0=H\left[y ; u_{0}\right]-H\left[u_{0} ; y\right] & =\int_{a}^{b} y S f d x-\int_{a}^{b} u_{0} S g d x \\
& =\int_{a}^{b} y S f d x,
\end{aligned}
$$

in view of (5.9) and the fact that $g$ is a vector of $L$. This completes the proof of Theorem 5.4 .

The above result for $H$-definitely self-adjoint systems is somewhat weaker than the corresponding result for definitely self-adjoint systems (see Bliss [2, Theorem 2.3]). Formally, this is true because the permanent convergence of the second series of (5.8) does not imply that the constant term $W_{0}$ of this series is equal to zero; the failure to obtain this latter result is in turn a consequence of the fact that we do not have an inequality of the form (5.6) for $\mu=0$. If the convergence of the second series of (5.8) were to imply the vanishing of $W_{0}$, by the argument used above we could proceed to show that the hypotheses of the above theorem imply the relation (5.1) for arbitrary vectors of the space $L$ instead of merely for the vectors belonging to $L^{2}$. That the result of the above theorem cannot in general be thus strengthened, however, is shown by the following example.

Consider the system

$$
\begin{aligned}
y_{1}^{\prime} & =0, & y_{2}^{\prime} & =-\lambda b(x) y_{1}^{\prime}, \\
y_{1}(0)-y_{2}(0) & =0, & y_{1}(1)+y_{2}(1) & =0,
\end{aligned}
$$

where $b(x)$ is a continuous function not identically zero on $0 \leqq x \leqq 1$, and such that

$$
\int_{0}^{1} b(x) d x=0
$$

It may readily be verified that this system is $H$-definitely self-adjoint with the matrix

$$
T \equiv\left\|\begin{array}{rr}
0 & 1 \\
-1 & 0
\end{array}\right\|,
$$

and, moreover, this system has no characteristic values. For this system, therefore, the condition of Theorem 5.4 that (5.1) hold for every characteristic solution imposes no additional restriction on a vector $f \equiv\left(f_{1}(x), f_{2}(x)\right)$ with continuous components. Now

$$
y_{1}(x) \equiv 1, \quad y_{2}(x)=\dot{1}-\left(2 / \int_{0}^{1} b^{2}(t) d t\right) \int_{0}^{x} b^{2}(t) d t
$$


is a vector of the space $L$ for this problem, and for this particular $y$ we have

$$
\int_{0}^{1} f S y d x=\int_{0}^{1} f_{1}(x) b(x) y_{1}(x) d x=\int_{0}^{1} f_{1}(x) b(x) d x .
$$

For certain continuous functions $f_{1}(x)$, in particular, for $f_{1}(x)=b(x)$, this expression is different from zero. Thus we see that in the statement of Theorem 5.4 the phrase "every vector $y$ of $L^{2}$ " cannot in general be replaced by "every vector $y$ of $L . "$

We shall now proceed to establish as corollaries.to the above theorem certain related results.

CoROllary 1. If the system (2.1) is H-definitely self-adjoint and $f(x)$ is a vector of the corresponding space $L$ for which condition (5.1) is satisfied by every characteristic solution $y$ of the system, then this condition is also satisfied by every vector $y$ of $L$; in particular, $\int_{a}^{b} f S f d x=0$.

Let $g(x)$ be a vector with continuous components such that $\mathcal{L}[f]=B g$, $s[f]=0$. For a characteristic solution $y$ corresponding to a characteristic value $\lambda$ we have

$$
\int_{a}^{b} y S g d x=H[y ; f]=H[f ; y]=\lambda \int_{a}^{b} f S y d x,
$$

and thus the condition that $f$ satisfies (5.1) with every characteristic solution $y$ implies that the vector $g$ satisfies a like condition. It then follows from Theorem 5.4 that $\int_{a}^{b} y^{*} S g d x=0$ for arbitrary vectors $y^{*}$ of $L^{2}$. Now for an arbitrary vector $y$ of $L$ let $y^{*}$ denote the vector of $L^{2}$ such that $\mathcal{L}\left[y^{*}\right]=B y, s\left[y^{*}\right]=0$. Then

$$
0=\int_{a}^{b} y^{*} S g d x=H\left[y^{*} ; f\right]=H\left[f ; y^{*}\right]=\int_{a}^{b} f S y d x
$$

so that the conditions of the corollary imply (5.1) for arbitrary vectors $y$ of $L$. Since $f(x)$ belongs to $L$, we have, in particular, $\int_{a}^{b} f S f d x=0$.

COROLlary 2. If the system (2.1) is $H$-definitely self-adjoint and $f(x)$ is a vector of the corresponding space $L^{2}$ for which condition (5.1) is satisfied by every characteristic solution $y$ of the system, then $B(x) f(x) \equiv 0$ on the interval ab.

Let $g(x)$ be a vector of $L$ such that $\mathcal{L}[f]=B g, s[f]=0$. Then by an argument similar to that used in the proof of Corollary 1 we have $\int_{a}^{b} y S g d x=0$ for every characteristic solution $y$ and hence, by Theorem 5.4, this condition also holds for arbitrary vectors $y$ of $L^{2}$. In particular, for $y=f$ we have

$$
0=\int_{a}^{b} f S g d x=H[f]
$$

and in view of (iii)' we have $B f \equiv 0$ on $a b$. 
Corollary 3.' If for an H-definitely self-adjoint system the condition $B(x) y(x) \equiv 0$ on ab holds for a vector $y$ of $L$ if and only if $y(x) \equiv 0$ on this interval, then if the components of $f(x)$ are continuous and condition (5.1) is satisfied by every characteristic solution of the system it follows that $B(x) f(x) \equiv 0$ on the interval $a b$.

In the proof of Theorem 5.4 it was established that the vector $u_{1}$ of $L$ defined by (5.3) satisfies $B u_{1} \equiv 0$ on $a b$. Under the strengthened hypotheses of the corollary we consequently have $u_{1} \equiv 0$, and it then follows from (5.4) for $\mu=1$ that $B u_{0} \equiv 0$ on $a b$. As $u_{0}$ is also a vector of $L$ it in turn results that $u_{0} \equiv 0$, and hence $B(x) f(x) \equiv 0$ on $a b$ by equation (5.4) for $\mu=0$.

For an $H$-definitely self-adjoint system the additional hypothesis of the above corollary is clearly equivalent to the following: $H[y]>0$ for every nonidentically vanishing vector $y$ of $L$.

6. Existence of characteristic values. In general an $H$-definitely self-adjoint system (2.1) does not possess an infinity of characteristic values. In particular, the example (5.11) of the preceding section illustrates the possibility that such a system may have no characteristic values. It is also easy to construct examples of such systems that have only a finite number of characteristic values. We shall, therefore, consider in this section the possible character of the totality of characteristic values of an $H$-definitely self-adjoint system.

Since for such a system the characteristic values are the zeros of a permanently convergent power series, and the index of each characteristic value is equal to its multiplicity, it follows that there can exist at most a denumerable infinity of characteristic values. Let $\left\{y_{\mu}, \lambda_{\mu}\right\},(\mu=1,2, \cdots)$, denote a maximal set of linearly independent characteristic solutions and corresponding characteristic values, the former chosen orthonormal in the sense that

$$
\int_{a}^{b} y_{\mu} S y_{\nu} d x=\delta_{\mu \nu} \frac{\left|\lambda_{\mu}\right|}{\lambda_{\mu}}, \quad \mu, \nu=1,2, \cdots,
$$

where $\delta_{\mu \nu}=0$ if $\mu \neq \nu, \delta_{\mu \mu}=1$. Such a choice is possible in view of Theorem 5.2.

Theorem 6.1. A necessary and sufficient condition that an $H$-definitely selfadjoint system have at least $k$ linearly independent characteristic solutions is that the quadratic functional $H[y]$ be positive definite on a linear subspace of $L^{2}$ of dimension $k$; that is, that there exist vectors $f_{\mu}(x),(\mu=1, \cdots, k)$, of $L^{2}$ such that for arbitrary constants $\left(d_{1}, \cdots, d_{k}\right) \neq(0, \cdots, 0)$ the vector $f(x)=f_{1}(x) d_{1}+\cdots$ $+f_{k}(x) d_{k}$ renders $H[f]>0$.

For suppose that $y_{\mu}(x),(\mu=1, \cdots, k)$, are linearly independent characteristic solutions of such a system, and that these solutions are chosen orthonormal in the sense of (6.1). If $\lambda_{\mu}$ denote the characteristic value corresponding to $y_{\mu}$, then for $f_{\mu}=y_{\mu}$ and arbitrary constants $\left(d_{1}, \cdots, d_{k}\right) \neq(0, \cdots, 0)$ we 
have for each vector $f=f_{1} d_{1}+\cdots+f_{k} d_{k}$ that $H[f]=\left|\lambda_{1}\right| d_{1}^{2}+\cdots+\left|\lambda_{k}\right| d_{k}^{2}>0$. Hence the condition of the theorem is necessary.

In order to prove the sufficiency of the theorem, suppose that there exists a linear subspace of $L^{2}$ determined by vectors $f_{1}, \cdots, f_{k}$ on which $H[y]$ is positive definite, while the system (2.1) has fewer than $k$ linearly independent characteristic solutions. It would then follow that there exists a set of constants $d_{1}, \cdots, d_{k}$ not all zero and such that the vector $f=f_{1} d_{1}+\cdots+f_{k} d_{k}$ satisfies equation (5.1) with every characteristic solution $y$. Since $f$ belongs to $L^{2}$, it is then a consequence of Corollary 2 to Theorem 5.4 that $B f \equiv 0$, and hence $H[f]=0$ also, contrary to the assumption of the positive definite character of $H[y]$ on the linear subspace of $L^{2}$ determined by $f_{1}, \cdots, f_{k}$. Hence the condition is also sufficient.

We shall now give a particular sufficient condition for an $H$-definitely self-adjoint system to have an infinity of characteristic values. This condition has application for the special boundary value problem of $\$ 10$. Suppose that the matrices $A(x)$ and $B(x)$ satisfy the following condition.

(v) There is a subinterval $a_{1} b_{1}, a<a_{1}<b_{1}<b$, of $a b$ such that if $a_{1}^{\prime}, b_{1}^{\prime}$ are arbitrary values satisfying $a_{1} \leqq a_{1}^{\prime}<b_{1}^{\prime} \leqq b_{1}$, then there exists a vector $g$ of $L$ and associated $y$ of $L^{2}$ satisfying $\mathcal{L}[y]=B g, B y \not \equiv 0$ on $a_{1}^{\prime} b_{1}^{\prime}$, whereas $y \equiv 0$ outside the given interval $a_{1}^{\prime} b_{1}^{\prime}$.

THEOREM 6.2. If an H-definitely self-adjoint system satisfies condition (v), then this system has infinitely many characteristic values.

For consider an interval $a_{1} b_{1}$ on which the condition $(v)$ is satisfied, and for a given integer $k$ divide $a_{1} b_{1}$ into $k$ non-overlapping subintervals $\Delta_{1}, \cdots, \Delta_{k}$. Let $y=f_{\mu}$ denote a vector of $L^{2}$ satisfying the conditions of $(\mathrm{v})$ relative to $\Delta_{\mu},(\mu=1, \cdots, k)$. Since $B f_{\mu} \not \equiv 0$ on $\Delta_{\mu}$, and $f_{\mu} \equiv 0$ outside this interval, it follows readily that $H\left[f_{\mu}\right]>0, H\left[f_{\mu} ; f_{\nu}\right]=0$ for $\mu \neq \nu,(\mu ; \nu=1, \cdots, k)$. Consequently, for each $f=f_{1} d_{1}+\cdots+f_{k} d_{k}$ we have $H[f]=H\left[f_{1}\right] d_{1}^{2}+\cdots$ $+H\left[f_{k}\right] d_{k}^{2}$, and by Theorem 6.1 the system (2.1) has at least $k$ linearly independent characteristic solutions. Since $k$ may be chosen arbitrarily, such a system has infinitely many characteristic values.

Corresponding to a vector $f$ we shall denote by $c_{\mu}[f]$ the Fourier coefficients

$$
c_{\mu}[f]=\frac{\left|\lambda_{\mu}\right|}{\lambda_{\mu}} \int_{a}^{b} f S y_{\mu} d x, \quad \mu=1,2, \cdots .
$$

Clearly these coefficients are well-defined for a vector $f$ whose components are merely in tegrable on $a b$.

LemMA 6.1. If $\left\{y_{\mu}, \lambda_{\mu}\right\},(\mu=1,2, \cdots)$, denote a maximal set of linearly independent characteristic solutions and corresponding characteristic values for an $H$-definitely self-adjoint system (2.1), the former orthonormal in the sense of (6.1), then for an arbitrary vector $f$ of $L$, 


$$
\sum_{\mu}\left|\lambda_{\mu}\right| c_{\mu}^{2}[f] \leqq H[f]
$$

If $f$ belongs to $L$, then for arbitrary integers $k$ the vector $f-\sum_{\mu_{\Perp} k} y_{\mu}(x) c_{\mu}[f]$ is also in $L$, and

$$
0 \leqq H\left[f-\sum_{\mu \leqq k} y_{\mu} c_{\mu}\right]=H[f]-\sum_{\mu \leqq k}\left|\lambda_{\mu}\right| c_{\mu}^{2}[f] .
$$

7. Definitely self-adjoint systems. In this section we shall consider systems (2.1) that are definitely self-adjoint in the sense of Bliss. A maximal set of linearly independent characteristic solutions and associated characteristic values for such a system will again be denoted by $\left\{y_{\mu}(x), \lambda_{\mu}\right\},(\mu=1,2, \cdots)$; moreover, we shall assume that the former are chosen orthonormal in the sense that

$$
\int_{a}^{b} y_{\mu} S y_{\nu} d x=\delta_{\mu \nu}, \quad \quad \mu, \nu=1,2, \cdots
$$

We also write

$$
e_{\mu}[f]=\int_{a}^{b} f S y_{\mu} d x, \quad \quad \mu=1,2, \cdots,
$$

for the Fourier coefficients of a vector $f(x)$. It then follows from Theorem 3.1 of Bliss [2] that for an arbitrary vector $f$ of $L$ the series

$$
\phi(x)=\sum_{\mu} y_{\mu}(x) e_{\mu}[f]
$$

converges absolutely and uniformly on $a b$; moreover, $B(x)[f(x)-\phi(x)] \equiv 0$ on this interval.

THEOREM 7.1. If (2.1) is definitely self-adjoint, then for arbitrary vectors $f$ of $L$ we have

$$
H[f]=\sum_{\mu} \lambda_{\mu} e_{\mu}^{2}[f], \quad \int_{a}^{b} f S f d x=\sum_{\mu} e_{\mu}^{2}[f] .
$$

The uniform convergence of the series (7.3) permits the evaluation of $H[f]$ as

$$
\begin{aligned}
H[f]=\int_{a}^{b} f S g d x & =\int_{a}^{b} g S f d x \\
& =\int_{a}^{b} g S \phi d x \\
& =\sum_{\mu} e_{\mu}[g] e_{\mu}[f] \\
& =\sum_{\mu} \lambda_{\mu} e_{\mu}^{2}[f],
\end{aligned}
$$


where $g$ is a vector such that $\mathcal{L}[f]=B g$; the last relation above is a consesequence of the readily established equality $e_{\mu}[g]=\lambda_{\mu} e_{\mu}[f]$. Similarly

$$
\int_{a}^{b} f S f d x=\int_{a}^{b} f S \phi d x=\sum_{\mu} e_{\mu}^{2}[f] .
$$

THEOREM 7.2. Suppose that (2.1) is definitely self-adjoint and that the characteristic values of this system are bounded below; moreover, let the set $\left\{y_{\mu}(x), \lambda_{\mu}\right\}$ be so ordered that $\lambda_{1} \leqq \lambda_{2} \leqq \ldots$. If $C_{1}$ denote the totality of vectors $f$ of $L$ satisfying $\int_{a}^{b} f S f d x=1$ and $C_{1}$ is nonvacuous, then $\lambda_{1}$ is the minimum of $H[f]$ in this class; moreover, this minimum is attained by a particular $f$ of $C_{1}$ if and only if $f=Y_{1}(x)+\Phi_{1}(x)$, where $Y_{1}$ is a characteristic solution for $\lambda=\lambda_{1}$ and $\Phi_{1}$ is a vector of $L$ such that $B \Phi_{1} \equiv 0$. In general, if $\lambda_{1}, \cdots, \lambda_{m-1}$ exist, denote by $C_{m}$ the totality of vectors $f$ of $L$ satisfying

$$
\int_{a}^{b} f S f d x=1, \quad e_{\mu}[f] \equiv \int_{a}^{b} f S y_{\mu} d x=0, \quad \mu=1, \cdots, m-1 .
$$

If this class is nonvacuous, then $\lambda_{m}$ exists and is the minimum of $H[f]$ in $C_{m}$; moreover, this minimum is attained by a particular $f$ of $C_{m}$ if and only if $f=Y_{m}(x)+\Phi_{m}(x)$, where $Y_{m}$ is a characteristic solution for $\lambda=\lambda_{m}$ and $\Phi_{m}$ is a vector of $L$ such that $B \Phi_{m} \equiv 0$ on $a b$.

The relations (7.4) clearly imply $H[f] \geqq \lambda_{1}$ in $C_{1}$ whenever this class is nonvacuous; furthermore, if $\lambda_{1}=\lambda_{2}=\cdots=\lambda_{q}<\lambda_{q+1}$, then the equality sign holds if and only if $e_{\mu}[f]=0,(\mu=q+1, \cdots)$. If $Y_{1}(x)=y_{1}(x) e_{1}[f]+\cdots+y_{q}(x) e_{q}[f]$, then $\Phi_{1}(x)=f(x)-Y_{1}(x)$ belongs to $L$ and $e_{\mu}\left[\Phi_{1}\right]=0,(\mu=1,2, \cdots)$. Hence by the second equation of (7.4), and the definiteness of $S$ we have $B \Phi_{1} \equiv 0$ on $a b$ (see also Bliss [2, Corollary 2.2]). In general, if $\lambda_{1}, \cdots, \lambda_{m-1}$ exist and the class $C_{m}$ is nonvacuous, it again follows from (7.4) that $\lambda_{m}$ must exist and $H[f] \geqq \lambda_{m}$ in this class. Moreover, if $\lambda_{m}=\lambda_{m+1}=\cdots=\lambda_{m+p}<\lambda_{m+p+1}$, then the equality sign holds if and only if $e_{\mu}[f]=0$ for $\mu>m+p$. If we now define $Y_{m}(x)=y_{m}(x) e_{m}[f]+\cdots+y_{m+p}(x) e_{m+p}[f]$, then $\Phi_{m}=f-Y_{m}$ is a vector of $L$ such that $e_{\mu}\left[\Phi_{m}\right]=0,(\mu=1,2, \cdots)$. Then, as above, it follows that $B \Phi_{m} \equiv 0$ on $a b$.

Theorem 7.3. If (2.1) is definitely self-adjoint and its characteristic values are either bounded below or above, then without loss of generality this system may be taken to be $H$-definitely self-adjoint; moreover, in this case $B B \equiv 0$ on $a b$, and the rank of $B(x)$ does not exceed $[n / 2]$ at any point of this interval.

Suppose that the characteristic values are bounded below, and let $\lambda_{0}$ be a number less than the smallest characteristic value, $\lambda_{1}$. Then for an arbitrary vector $f$ of $L$ the functional

$$
H\left[f: \lambda_{0}\right]=\int_{a}^{b} f \tilde{T}\left(\mathcal{L}[f]-\lambda_{0} B f\right) d x=H[f]-\lambda_{0} \int_{a}^{b} f S f d x
$$


may be written, in view of (7.4), as

$$
H\left[f: \lambda_{0}\right]=\sum_{\mu}\left(\lambda_{\mu}-\lambda_{0}\right) e_{\mu}^{2}[f] .
$$

Consequently, for $f$ belonging to $L$ we have $H\left[f: \lambda_{0}\right] \geqq 0$, and the equality sign holds if and only if $e_{\mu}[f]=0,(\mu=1,2, \cdots)$. For a definitely self-adjoint system, however, this condition implies $B f \equiv 0$ on $a b$ in view of the second equation of (7.4) (see also Bliss [2, Corollary 2.2]). The replacement of $H[f]$ by $H\left[f: \lambda_{0}\right]$ is equivalent to a linear change of parameter in the boundary value problem (2.1). Hence for a definitely self-adjoint problem whose characteristic values are bounded below we may without loss of generality assume that the functional $H[y]$ satisfies the definiteness property (iii)'; that is, that the system is $H$-definitely self-adjoint. By Theorem 4.3 it then follows that $B B \equiv 0$ and the rank of this matrix does not exceed $[n / 2]$ on $a b$.

In case the characteristic values of (2.1) are bounded above then the replacement of $\lambda$ by $-\lambda$, or the equivalent replacement of $B(x)$ by $-B(x)$, transforms the given system into one whose characteristic values are bounded below. The original system being definitely self-adjoint with $T$ implies that the new system is definitely self-adjoint with $-T$. Hence, by a linear change of parameter and the replacement of $T$ by $-T$ the given system is reducible to one which is $H$-definitely self-adjoint and the results of the theorem follow from the preceding case.

In this connection, it is worthwhile to point out that certain specific representations of "equivalent" boundary value problems may have individual advantages. For example, consider the boundary value problem $y^{\prime \prime}+\lambda y=0$, $y(0)=0=y(\pi)$. A maximal set of linearly independent characteristic functions and associated characteristic values is $\left\{\sin n x, n^{2}\right\},(n=1,2, \cdots)$. If we write this problem as $y_{1}^{\prime}=y_{2}, y_{2}^{\prime}=-\lambda y_{1}, y_{1}(0)=0=y_{1}(\pi)$, then this system is definitely self-adjoint and also $H$-definitely self-adjoint with the matrix (5.13). On the other hand, $y_{1}^{\prime}=\rho y_{2}, y_{2}^{\prime}=-\rho y_{1}, y_{1}(0)=0=y_{1}(\pi)$ is "equivalent" to the given problem by setting $\lambda=\rho^{2}$. This latter system is definitely self-adjoint with (5.13), but is clearly not $H$-definitely self-adjoint with this or any other matrix $T$ since the corresponding matrix $B(x)$ is nonsingular.

The following result is an immediate consequence of Theorem 7.3.

TheOREM 7.4. If (2.1) is definitely self-adjoint and $B(x) B(x) \not \equiv 0$ on $a b$, then this system has infinitely many negative, and also infinitely many positive, characteristic values.

This theorem contains as a very special case the result of Theorem 4.3 of Reid [10]. To see this, suppose that $B(x)$ has constant rank $n-m$ on $a b$ and denote by $\pi_{i}=\pi_{i \alpha}(x),(\alpha=1, \cdots, m)$, linearly independent solutions of the equations $B(x) \pi=0$. From (2.4) it follows that $\tilde{T}^{-1} \tilde{B} \equiv B T^{-1}$, and hence the rank of $\left\|\pi_{i \alpha}(x) T_{k l}^{-1}(x) B_{j k}(x)\right\|$ is the same as the rank of $\left\|\pi_{i \alpha}(x) B_{i k}(x) T_{k j}^{-1}(x)\right\|$, 
which in turn is equal to the rank of $\left\|\pi_{i \alpha}(x) B_{i j}(x)\right\|$. Now the rank of this latter matrix is clearly equal to $m$ if $B B \equiv 0$, and hence the hypotheses of Theorem 4.3 of [10], which demand that the rank of this matrix exceed $m$ at some point $x_{0}$ of $a b$, require that $B B \not \equiv 0$ on $a b$. It is also to be noted that in the above referred to theorem of [10] it was not proved that the system had infinitely many characteristic values of each algebraic sign, but simply that the system had infinitely many characteristic values under the stronger hypotheses there stated.

In view of relations (7.4) we have the following result.

Theorem 7.5. A definitely self-adjoint system (2.1) has at least $k$ characteristic values if and only if $\int_{a}^{b} f S f d x$ is positive definite on a linear subspace of $L$ of dimension $k$. Moreover, for a given constant $\lambda_{0}$ such a system has at least $k$ characteristic values greater [less] than $\lambda_{0}$ if and only if the functional $H\left[f: \lambda_{0}\right]$ is positive [negative] definite on a linear subspace of $L$ of dimension $k$.

In the case of a definitely self-adjoint system for which the matrix $B(x)$ has constant rank on $a b$ the first part of this theorem was deduced by Reid [10, Theorem 4.1] from known results for an auxiliary problem associated with the calculus of variations. Analogues of the above theorem for $H$-definitely self-adjoint systems are contained in Theorem 6.1 and Theorem 9.3.

8. A special definitely self-adjoint system. Suppose now that the boundary value problem (2.1) satisfies conditions (i), (ii) and (iv) of $\$ 2$ with a matrix $T(x)$. In this section we shall consider the associated system

$$
\mathcal{L}[y]=\lambda B_{1}(x) y, \quad s[y]=0,
$$

where $B_{1}(x)=B(x) \tilde{T}(x) B(x)=B(x) S(x)$. This problem is seen to be definitely self-adjoint with the same matrix $T(x)$. In the first place, in order to show that (8.1) is self-adjoint with $T$ it remains only to show that $T B_{1}+\tilde{B}_{1} T \equiv 0$, and this is true since $T B \tilde{T} B+\tilde{B} T \tilde{B} T \equiv(T B+\tilde{B} T) S \equiv 0$ by (2.3). If we set $S_{1}(x)=\tilde{T}(x) B_{1}(x)=S(x) S(x)$, clearly conditions (ii) and (iii) are satisfied by $S_{1}$. Finally, since $B_{1} y \equiv 0$ implies $y \tilde{T} B_{1} y \equiv y S S y \equiv 0$, and hence $S y \equiv 0$ and $B y \equiv 0$, condition (iv) for (2.1) implies the corresponding condition for (8.1).

Since a definitely self-adjoint problem has at most a denumerable infinity of characteristic values, for the consideration of (8.1) one may assume without loss of generality that $\lambda=0$ is not a characteristic value of this system. If this condition is not true for the problem as written, it is attainable by a linear change of parameter. We shall make this assumption in the following discussion.

If $y$ is a characteristic solution of (8.1) corresponding to a value $\lambda$, set $u(x)=S(x) y(x)$. In view of condition (iv) for (8.1) we have $u \neq 0$ on $a b$. Then $\mathcal{L}[y]=\lambda B u, s[y]=0$, and if $G(x, t)$ denotes the Green's matrix for the incompatible system $\mathcal{L}[y]=0, s[y]=0$, we have

$$
y(x)=\lambda \int_{a}^{b} K(x, t) u(t) d t
$$


where, as in $\S 4, K(x, t)=G(x, t) B(t)$. In particular, it then follows that $u(x)$ is a characteristic solution, for this same value of $\lambda$, of the linear vector integral equation

$$
u(x)=\lambda \int_{a}^{b} K_{1}(x, t) u(t) d t,
$$

where, again as in $\S 4$, we have written $K_{1}(x, t)=S(x) K(x, t)$. It also follows from the comment after equation (4.4) that $K_{1}(x, t)=\tilde{K}_{1}(t, x)$, and hence (8.2) is of the type covered by the Hilbert-Schmidt theory of linear integral equations. Conversely, if $u$ is a characteristic solution of (8.2) for a value $\lambda$, and $y$ is defined as the corresponding unique solution of $\mathcal{L}[y]=\lambda B u, s[y]=0$, it follows that $u(x)=S(x) y(x)$ and $y$ is a characteristic solution of (8.1) for the same value of $\lambda$. Hence, there is complete equivalence between the boundary value problem (8.1) and the integral equation (8.2).

We shall denote by $\left\{y_{\sigma}, \Lambda_{\sigma}\right\},(\sigma=1,2, \cdots)$, a maximal set of linearly independent characteristic solutions and corresponding characteristic values of (8.1), the former chosen orthonormal in the sense that

$$
\int_{a}^{b} y_{\sigma} S_{1} y_{\tau} d x=\delta_{\sigma \tau}, \quad \sigma, \tau=1,2, \cdots .
$$

Correspondingly, $\left\{u_{\sigma}=S y_{\sigma}, \Lambda_{\sigma}\right\}$ is a maximal set of linearly independent characteristic solutions and corresponding characteristic values of (8.2) satisfying $\int_{a}^{b} u_{\sigma} u_{\tau} d x=\delta_{\sigma \tau}$.

Finally, if $g(x)$ is a vector whose components are continuous (or of Lebesgue integrable square) on $a b$, and if $f$ is defined by the system $\mathcal{L}[f]=B g$, $s[f]=0$, it follows from (4.3) that

$$
H[f]=\int_{a}^{b} \int_{a}^{b} g(x) K_{1}(x, t) g(t) d x d t .
$$

It is to be emphasized that the above defined vector $f$ belongs to the linear vector space $L$ for the problem (2.1), but not necessarily to the corresponding space $L_{1}$ for the problem (8.1), since this latter space contains vectors $f$ which satisfy with associated vectors $g$ the differential system

$$
\mathcal{L}[f]=B S g, \quad s[f]=0 .
$$

In case the matrix $B$ is nonsingular on $a b$ the space $L_{1}$ is seen to be identical with $L$. However, since in general $L_{1}$ is a subspace of $L$ and $B_{1} y \equiv 0$ on $a b$ if and only if $B y \equiv 0$ on this interval, the condition that (2.1) be $H$-definitely self-adjoint clearly implies that $(8: 1)$ is also $H$-definitely self-adjoint.

The results of Bliss [2], and those of the preceding section, give properties of the particular definitely self-adjoint system (8.1) on the space $L_{1}$. We wish, however, to go further and obtain properties of this system on the space $L$ corresponding to the boundary value problem (2.1). As pointed out above, 
one may always by a linear change of parameter, replacing $\lambda$ by a suitable $\lambda+\lambda_{0}$, insure for (8.1) that $\lambda=0$ is not a characteristic value of this system. Now this change of parameter is equivalent to replacing $A(x)$ by

$$
A(x)+\lambda_{0} B(x) S(x) \text {. }
$$

Before proceeding further, it is to be emphasized that the space $L$, as defined in $\$ 2$, is invariant under this operation. This results from the fact that for a given vector $y$ whose components are of class $C^{1}$ the vector differential equation $y^{\prime}-A y=B g$ is equivalent to the equation $y^{\prime}-A y-\lambda_{0} B S y=B g_{1}$ by the transformation $g_{1}=g-\lambda_{0} S y$.

Corresponding to a given vector $f$, we set

$$
d_{\sigma}[f]=\int_{a}^{b} f S_{1} y_{\sigma} d x, \quad \delta_{\sigma}[f]=\int_{a}^{b} f S y_{\sigma} d x=\int_{a}^{b} f u_{\sigma} d x, \quad \sigma=1,2, \cdots ;
$$

clearly these coefficients are well-defined if the components of $f$ are integrable on $a b$. Since the vectors $u_{\sigma}$ are orthonormal, the following result is an immediate consequence of Bessel's inequality.

LEMma 8.1. If the components of $g(x)$ are of integrable square on $a b$, then the series $\sum_{\sigma} \delta_{\sigma}^{2}[g]$ converges and

$$
\sum_{\sigma} \delta_{\sigma}^{2}[g] \leqq \int_{a}^{b} g g d x
$$

LEMMA 8.2. The series

$$
\sum_{\sigma}\left\{\frac{y_{i \sigma}(x)}{\Lambda_{\sigma}}\right\}^{2}, \quad i=1,2, \cdots, n,
$$

converge on $a b$; moreover, if $\sum_{\sigma} \delta_{\sigma}^{2}<+\infty$, the vector series

$$
\sum_{\sigma} \frac{y_{\sigma}(x)}{\Lambda_{\sigma}} \delta_{\sigma}
$$

converges absolutely and uniformly on this interval.

Since

$$
\frac{y_{\sigma}(x)}{\Lambda_{\sigma}}=\int_{a}^{b} K(x, t) u_{\sigma}(t) d t
$$

it follows that for a fixed value of $x$ each row of $K(x, t)$ is a vector satisfying the conditions of Lemma 8.1. Hence the series (8.6) converges; moreover, in view of (8.5), there clearly exists a constant $\kappa$ such that sum of the series (8.6) does not exceed $\kappa$ in value on $a b$. If $\sum_{\sigma} \delta_{\sigma}^{2}<+\infty$, the absolute and uniform convergence of (8.7) on this interval is a consequence of the inequalities 


$$
\begin{aligned}
\sum_{\sigma=N}^{N+h}\left|\frac{y_{i \sigma}(x)}{\Lambda_{\sigma}} \delta_{\sigma}\right| & \leqq\left[\sum_{\sigma=N}^{N+h}\left\{\frac{y_{i \sigma}(x)}{\Lambda_{\sigma}}\right\}^{2}\right]^{1 / 2} \cdot\left[\sum_{\sigma=N}^{N+h} \delta_{\sigma}^{2}\right]^{1 / 2} \\
& \leqq \kappa\left[\sum_{\sigma=N}^{N+h} \delta_{\sigma}^{2}\right]^{1 / 2} \cdot
\end{aligned}
$$

THEOREM 8.1. For an arbitrary vector $f$ of $L$ the series

$$
\phi(x)=\sum_{\sigma} y_{\sigma}(x) d_{\sigma}[f]
$$

converges absolutely and uniformly on ab; moreover, $B(x)[f(x)-\phi(x)] \equiv 0$ on this interval. For an arbitrary vector $h(x)$ whose components are integrable on $a b$,

$$
\int_{a}^{b} h S f d x=\sum_{\sigma} \delta_{\sigma}[h] d_{\sigma}[f]
$$

in particular,

$$
\begin{aligned}
\int_{a}^{b} f S_{1} f d x & =\sum_{\sigma} d_{\sigma}^{2}[f], \\
H[f] & =\sum_{\sigma} \Lambda_{\sigma} d_{\sigma}^{2}[f] .
\end{aligned}
$$

If $\mathcal{L}[f]=B g, s[f]=0$, it follows from (8.1) that $\Lambda_{\sigma} d_{\sigma}[f]=\delta_{\sigma}[g]$, $(\sigma=1,2, \cdots)$, and the absolute and uniform convergence of (8.8) is a consequence of Lemmas 8.1 and 8.2. Clearly, $\psi(x) \equiv S(x)[f(x)-\phi(x)]$ satisfies $\delta_{\sigma}[\psi]=0,(\sigma=1,2, \cdots)$. We will now show that also

$$
\int_{a}^{b} K_{1}(x, t) \psi(t) d t=0
$$

Let $f^{*}$ be the solution of $\mathcal{L}\left[f^{*}\right]=B_{1} f, s\left[f^{*}\right]=0$. Then by Theorem 3.1 of Bliss [2] the series $\phi^{*}(x)=\sum_{\sigma} y_{\sigma}(x) d_{\sigma}\left[f^{*}\right]$ converges absolutely and uniformly on $a b$, and $B_{1}\left[f^{*}-\phi^{*}\right] \equiv 0$ on this interval. This latter condition, in view of the first paragraph of this section, implies $S\left[f^{*}-\phi^{*}\right] \equiv 0$. Consequently, since $\Lambda_{\sigma} d_{\sigma}\left[f^{*}\right]=d_{\sigma}[f],(\sigma=1,2, \cdots)$, we have

$$
0 \equiv S(x)\left[f^{*}(x)-\sum_{\sigma} \frac{y_{\sigma}(x)}{\Lambda_{\sigma}} d_{\sigma}[f]\right] \equiv \int_{a}^{b} K_{1}(x, t) \psi(t) d t,
$$

the latter relation being verified by direct computation. Finally, as

$$
\psi(x)=\int_{a}^{b} g(t) K_{1}(t, x) d t-\sum_{\sigma} u_{\sigma}(x) d_{\sigma}[f],
$$

it follows from (8.12) and $\delta_{\sigma}[\psi]=0,(\sigma=1,2, \cdots)$, that $\int_{a}^{b} \psi \psi d x=0$, and hence $\psi \equiv 0$ on $a b$. In particular, $0 \equiv \tilde{T}^{-1} S[f-\phi] \equiv B[f-\phi]$. 
Equations (8.9) and (8.10) are ready consequences of the relation $S f \equiv S \phi$ and the uniform convergence of the series $\phi$. Relation (8.11) in turn results from (8.9), the conditions $\Lambda_{\sigma} d_{\sigma}[f]=\delta_{\sigma}[g]$, and $H[f]=\int_{a}^{b} g S f d x$.

In view of (8.10) we also have the following result.

Corollary 1. A vector $f$ of $L$ satisfies $\int_{a}^{b} f S_{1} y d x=0$ with every characteristic solution $y$ of (8.1) if and only if $B f \equiv 0$ on $a b$.

Corollary 2.2 of Bliss [2], when applied to the system (8.1), would imply the result of the preceding corollary for vectors $f$ belonging to $L_{1}$, instead of to the space $L$.

CoROLlaRy 2. If the vector $f$ belongs to $L$, and $f^{*}$ in turn satisfies $\mathcal{L}\left[f^{*}\right]=B_{1} f$, $s\left[f^{*}\right]=0$, then $f^{*}(x)=\sum_{\sigma} y_{\sigma}(x) d_{\sigma}\left[f^{*}\right]$.

In view of the uniform convergence of the series (8.8) associated with $f$, and the relation $\Lambda_{\sigma} d_{\sigma}\left[f^{*}\right]=d_{\sigma}[f],(\sigma=1,2, \cdots)$, we have

$$
\begin{aligned}
f^{*}(x) & =\int_{a}^{b} K(x, t) S(t) f(t) d t \\
& =\sum_{\sigma}\left\{\int_{a}^{b} K(x, t) S(t) y_{\sigma}(t) d t\right\} d_{\sigma}[f] \\
& =\sum_{\sigma} \frac{y_{\sigma}(x)}{\Lambda_{\sigma}} d_{\sigma}[f] \\
& =\sum_{\sigma} y_{\sigma}(x) d_{\sigma}\left[f^{*}\right] .
\end{aligned}
$$

It is to be mentioned that the above relation $S f \equiv \sum_{\sigma} u_{\sigma} \delta_{\sigma}[S f]=\sum_{\sigma} u_{\sigma} d_{\sigma}[f]$ for a vector $f$ of $L$, and the subsequent proof of (8.10), (8.11), could have been taken directly from the Hilbert-Schmidt theory of integral equations. However, by the above treatment we have proved more; namely, the absolute and uniform convergence of the series (8.8) involving the characteristic solutions of the considered boundary value problem (8.1).

If the characteristic values of (8.1) are bounded below, for this particular problem the result of Theorem 7.2 may be strengthened in that the space $L$ for the problem (2.1) may essentially be substituted for the space $L_{1}$ belonging to (8.1). For suppose that the characteristic values are bounded below and that $\left\{y_{\sigma}, \Lambda_{\sigma}\right\}$ are so ordered that $\Lambda_{1} \leqq \Lambda_{2} \leqq \ldots$. If $\Gamma_{1}$ denote the totality of vectors $f$ of $L$ satisfying $\int_{a}^{b} f S_{1} f d x=1$ and $\Gamma_{1}$ is nonvacuous, it follows from (8.10), (8.11) that $\Lambda_{1}$ is the minimum of $H[f]$ in $\Gamma_{1}$; moreover, in view of Corollary 1 , it follows by an argument similar to that used in the proof of Theorem 7.2 that this minimum is attained by a particular $f$ if and only if $f=Y_{1}+\Phi_{1}$, where $Y_{1}$ is a characteristic solution of (8.1) for $\lambda=\Lambda_{1}$ and $\Phi_{1}$ is a vector of $L$ such that $B \Phi_{1} \equiv 0$. In general, if $\Lambda_{1}, \cdots, \Lambda_{m-1}$ exist for (8.1), let $\Gamma_{m}$ de- 
note the totality of vectors $f$ of $L$ satisfying $\int_{a}^{b} f S_{1} f d x=1, d_{\sigma}[f]=0$, $(\sigma=1, \cdots, m-1)$. If this class is nonvacuous, we have by a corresponding argument that $\Lambda_{m}$ exists and is the minimum of $H[f]$ in $\Gamma_{m}$; moreover, this minimum is attained by a particular $f$ of $\Gamma_{m}$ if and only if $f$ is of the form $Y_{m}+\Phi_{m}$, where $Y_{m}$ is a characteristic solution for $\lambda=\Lambda_{m}$ and $\Phi_{m}$ is a vector of $L$ satisfying $B \Phi_{m} \equiv 0$ on $a b$.

From the above Corollary 1 and equation (8.11) we deduce the following theorem.

THEOREM 8.2. A system (2.1) which satisfies conditions (i), (ii) and (iv) of §2 also satisfies condition (iii)', and is consequently $H$-definitely self-adjoint, if and only if the corresponding system (8.1) has no characteristic values $\Lambda$ satisfying $\Lambda \leqq 0$.

It is to be emphasized that there is no assurance under the conditions of this theorem that the system (8.1) shall have any characteristic values. For example, the system

$$
y_{1}^{\prime}=0, \quad y_{2}^{\prime}=-\lambda y_{1}, \quad y_{1}(0)=0=y_{2}(1)
$$

is not only definitely self-adjoint (Bliss [2, p. 427]), but also $H$-definitely self-adjoint with the matrix (5.13), whereas this system has no characteristic values. Moreover, the corresponding system (8.1) is identical with the given system and thus possesses no characteristic values.

If an $H$-definitely self-adjoint system has $k$ linearly independent characteristic solutions it is a consequence of Theorem 6.1 and formula (8.11) that the corresponding system (8.1) has at least $k$ linearly independent characteristic solutions. In general, however, when (2.1) is $H$-definitely self-adjoint the corresponding system (8.1) may have more linearly independent characteristic solutions than the original system. To illustrate this possibility, consider the example (5.11) where, as in $\S 5$, it is supposed that $\int_{0}^{1} b(t) d t=0$. The corresponding system (8.1) is

$$
\begin{aligned}
y_{1}^{\prime} & =0, & y_{2}^{\prime} & =-\Lambda b^{2}(x) y_{1}, \\
y_{1}(0)-y_{2}(0) & =0, & y_{1}(1)+y_{2}(1) & =0,
\end{aligned}
$$

and this system is seen to have the single characteristic value $\Lambda=2 / \int_{0}^{i} b^{2}(t) d t$ of index one, whereas the original system (5.11) has no characteristic values.

THEOREM 8.3. For an $H$-definitely 'self-adjoint system (2.1) there exists a constant $d>0$ such that the inequality

$$
\int_{a}^{b} f S_{1} f d x \leqq d H[f]
$$

holds for arbitrary vectors $f$ of $L$. 
If the corresponding system (8.1) admits of characteristic values, then in view of Theorem 8.2 and the minimizing properties of the characteristic values, inequality (8.13) holds for $d$ the reciprocal of the smallest characteristic value. If (8.1) possesses no characteristic values, then $B f \equiv 0$ on $a b$ for every vector $f$ of $L$, the two integrals appearing in (8.13) are individually zero, and in this case $d$ may be chosen as an arbitrary positive number.

Since for an $H$-definitely self-adjoint system (2.1) the elements of the matrix $K_{1}(x, t)$ are continuous on $a \leqq x, t \leqq b$, and the quadratic functional (4.3) is positive semi-definite for arbitrary vectors $g$, we have the following theorem of Mercer (Mercer [8]; also, for example, [3, p. 456]).

THEOREM 8.4. If (2.1) is $H$-definitely self-adjoint the series

$$
\sum_{\sigma} \frac{u_{i \sigma}(x) u_{j \sigma}(t)}{\Lambda_{\sigma}}, \quad i, j=1,2, \cdots, n,
$$

converges absolutely and uniformly on $a \leqq x, t \leqq b$ and has the sum $K_{1 i j}(x, t)$.

9. Further results for $H$-definitely self-adjoint systems. The conclusions of the previous section will now be used in the proof of additional results for an $H$-definitely self-adjoint problem (2.1). For such a problem let $\boldsymbol{C}_{\mathbf{1}}$ denote the totality of vectors $f$ of $L$ satisfying the condition

$$
\int_{a}^{b} f S f d x=1
$$

THEOREM 9.1. If for an $H$-definitely self-adjoint problem (2.1) the class $\boldsymbol{C}_{1}$ is nonvacuous, then this system possesses positive characteristic values; moreover, the smallest positive characteristic value is the minimum of $H[f]$ in the class $\boldsymbol{C}_{1}$.

If the class $C_{1}$ is nonvacuous, let $\lambda_{1}$ denote the greatest lower bound of $H[f]$ in this class; it then follows that

$$
H\left[f: \lambda_{1}\right] \equiv H[f]-\lambda_{1} \int_{a}^{b} f S f d x \geqq 0
$$

for arbitrary vectors $f$ of $L$. In view of relation (2.5) for a characteristic solution, there is clearly no positive characteristic value of (2.1) less than $\lambda_{1}$. Hence we have only to prove that $\lambda_{1}$ is a characteristic value.

Theorem 9.1 will be established by indirect argument. Let $f_{m} \equiv\left(f_{i m}\right)$, $(m=1,2, \cdots)$, be a sequence of vectors of $C_{1}$ such that $\lim _{m \rightarrow \infty} H\left[f_{m}\right]=\lambda_{1}$; on the assumption that $C_{1}$ is not empty such a sequence exists. Now suppose that $\lambda=\lambda_{1}$ is not a characteristic value; then there exist unique corresponding vectors $h_{m}(x)$ such that

$$
\mathcal{L}\left[h_{m}\right]-\lambda_{1} B h_{m}=B f_{m}, \quad s\left[h_{m}\right]=0, \quad m=1,2, \cdots .
$$


Moreover, if $G\left(x, t: \lambda_{1}\right)$ denote the Green's matrix for the incompatible system $\mathcal{L}[y]-\lambda_{1} B y=0, s[y]=0$, we have

$$
h_{m}(x)=\int_{a}^{b} H\left(x, t: \lambda_{1}\right) S(t) f_{m}(t) d t, \quad m=1,2, \cdots,
$$

where $H\left(x, t: \lambda_{1}\right)=G\left(x, t: \lambda_{1}\right) \tilde{T}^{-1}(t)$. By an elementary vector inequality,

$$
\operatorname{norm}\left\{h_{m}(x)\right\} \leqq \kappa \int_{a}^{b} \operatorname{norm}\left\{S(t) f_{m}(t)\right\} d t=\kappa \int_{a}^{b}\left[f_{m} S_{\mathrm{i}} f_{m}\right]^{1 / 2} d t
$$

for $\kappa$ a suitable constant depending only upon the bounds of the elements of $H\left(x, t: \lambda_{1}\right)$ on $a \leqq x, t \leqq b$. By the use of Schwarz' inequality and Theorem 8.3 it then follows that

$$
\operatorname{norm}\left\{h_{m}(x)\right\} \leqq \kappa\left\{(b-a) d H\left[f_{m}\right]\right\}^{1 / 2} .
$$

In particular, since $\left\{f_{m}\right\}$ is a minimizing sequence of $\boldsymbol{C}_{1}$, the sequence $\left\{H\left[f_{m}\right]\right\}$ is uniformly bounded and there exists a constant $\kappa_{1}$ such that

$$
\int_{a}^{b}\left[\operatorname{norm}\left\{h_{m}(x)\right\}\right]^{2} d x \leqq \kappa_{1}, \quad m=1,2, \cdots .
$$

Now set $g_{m}(x)=f_{m}(x)+c h_{m}(x),(m=1,2, \cdots)$, where $c$ is a real constant. Then $g_{m}$ is a vector of $L$, and

$$
\begin{aligned}
H\left[g_{m}: \lambda_{1}\right] & =H\left[f_{m}: \lambda_{1}\right]+2 c H\left[f_{m} ; h_{m}: \lambda_{1}\right]+c^{2} H\left[h_{m}: \lambda_{1}\right] \\
& =H\left[f_{m}: \lambda_{1}\right]+2 c+c^{2} \int_{a}^{b} h_{m} S f_{m} d x,
\end{aligned}
$$

in view of (9.2) and the fact that $f_{m}$ belongs to the class $\boldsymbol{C}_{1}$. Now

$$
\begin{aligned}
\left|\int_{a}^{b} h_{m} S f_{m} d x\right| & \leqq \int_{a}^{b}\left[\operatorname{norm}\left\{h_{m}\right\}\right] \cdot\left[\operatorname{norm}\left\{S f_{m}\right\}\right] d x \\
& \leqq \frac{1}{2} \int_{a}^{b}\left[h_{m} h_{m}+f_{m} S_{1} f_{m}\right] d x \\
& \leqq \frac{1}{2}\left(\kappa_{1}+d H\left[f_{m}\right]\right),
\end{aligned}
$$

by (9.4) and Theorem 8.3. Consequently, since $\left\{H\left[f_{m}\right]\right\}$ is uniformly bounded, there exists a constant $\kappa_{2}$ such that

$$
\left|\int_{a}^{b} h_{m} S f_{m} d x\right| \leqq \kappa_{2}, \quad m^{\prime}=1,2, \cdots .
$$

Now let $c$ be a value such that $2 c+c^{2} \kappa_{2}<0$; that is, $0>c>-2 / \kappa_{2}$. As 
$\lim _{m \rightarrow \infty} H\left[f_{m}: \lambda_{1}\right]=0$, for $m$ sufficiently large it follows that $H\left[g_{m}: \lambda_{1}\right]<0$, contrary to the definition of $\lambda_{1}$. Hence $\lambda_{1}$ is a characteristic value and the theorem is proved.

Let $C_{-1}$ denote the totality of vectors $f$ of $L$ satisfying

$$
\int_{a}^{b} f S f d x=-1
$$

If the matrix $B(x)$ is replaced by $-B(x)$, then the class $C_{-1}$ for the original problem corresponds to the class $C_{1}$ for the modified problem; clearly such a substitution does not affect the $H$-definite self-adjointness of the problem. Hence we have the following result.

COROLlARY. If for an $\mathrm{H}$-definitely self-adjoint problem the class $\boldsymbol{C}_{-1}$ is nonvacuous, then this system possesses negative characteristic values; moreover, if $\lambda_{-1}$ denote the largest negative characteristic value, then $-\lambda_{-1}$ is the minimum of $H[f]$ in the class $\boldsymbol{C}_{-1}$.

For convenience, in the remainder of this section we shall denote the totality of positive characteristic values of $(2.1)$ by $\left\{\lambda_{m}\right\},(m=1,2, \cdots)$, each repeated a number of times equal to its multiplicity and ordered so that $\lambda_{1} \leqq \lambda_{2} \leqq \cdots$. Similarly, $\left\{\lambda_{-m}\right\},(m=1,2, \cdots)$, denotes the totality of negative characteristic values, each repeated a number of times equal to its multiplicity and the set ordered so that $\lambda_{-1} \geqq \lambda_{-2} \geqq \cdots$. Corresponding to $\lambda_{m}, \lambda_{-m}$ we shall associate characteristic solutions $y_{m}, y_{-m}$, respectively, such that $\left\{y_{m}, y_{-m}\right\},(m=1,2, \cdots)$, is a maximal set of linearly independent solutions orthonormal in the sense of (6.1). Clearly either one, or both, of the sequences $\left\{y_{m}, \lambda_{m}\right\},\left\{y_{-m}, \lambda_{-m}\right\}$ may be vacuous or consist of only a finite number of characteristic values and associated characteristic solutions.

Using the above notation, if $\lambda_{1}, \cdots, \lambda_{8-1}$ exist we shall denote by $C_{s}$ the totality of vectors $f$ of $L$ such that

$$
\int_{a}^{b} f S f d x=1, \quad \int_{a}^{b} y_{m} S f d x=0, \quad m=1,2, \cdots, s-1 .
$$

Similarly, if $\lambda_{-1}, \cdots, \lambda_{-(s-1)}$ exist the class $C_{-8}$ is defined as the totality of vectors $f$ of $L$ satisfying

$$
\int_{a}^{b} f S f d x=-1, \quad \int_{a}^{b} y_{-m} S f d x=0, \quad m=1,2, \cdots, s-1 .
$$

THEOREM 9.2. If for an $H$-definitely self-adjoint system the class $\boldsymbol{C}_{8}\left[\boldsymbol{C}_{-8}\right]$ is nonvacuous, then the characteristic value $\lambda_{s}\left[\lambda_{-s}\right]$ exists; moreover, $\lambda_{s}\left[-\lambda_{-s}\right]$ is the minimum of $H[f]$ in the class $C_{s}\left[C_{-s}\right]$.

In view of the artifice used in deducing the above corollary, it suffices to restrict our attention to the case of positive characteristic values. The result 
of the theorem might be established by an argument similar to that utilized by the author [7] in proving a corresponding result for special bòndary value problems associated with the calculus of variations. However, the following method, which has also been used in considering accessory boundary problems of the calculus of variations, seems more elegant.

Consider the auxiliary boundary problem involving $n+2(s-1)$ variables $(y, u, v) \equiv\left(y_{i}, u_{\alpha}, v_{\alpha}\right),(i=1,2, \cdots, n ; \alpha=1,2, \cdots, s-1)$, and consisting of the differential equations and boundary conditions

$$
\begin{aligned}
y_{i}^{\prime}=A_{i j}(x) y_{j}+B_{i j}(x) y_{j \beta}(x) u_{\beta}+\lambda B_{i j}(x) y_{j} \\
u_{\alpha}^{\prime}=0 \\
v_{\alpha}^{\prime}=y_{i \alpha}(x) S_{i j}(x) y_{j} \\
M_{i j} y_{j}(a)+N_{i j} y_{j}(b)=0 \\
v_{\alpha}(a)=0 \\
v_{\alpha}(b)=0
\end{aligned}
$$

where the indices $\alpha, \beta$ range from 1 to $s-1$. If capital German letters denote the matrices of (9.5) corresponding to $A, B, M$ and $N$ of the system (2.1), we have

$$
\begin{aligned}
\mathfrak{A}=\left\|\begin{array}{lll}
A_{i j} & B_{i j} y_{j \beta} & 0_{i \beta} \\
0_{\alpha j} & 0_{\alpha \beta} & 0_{\alpha \beta} \\
y_{i \alpha} S_{i j} & 0_{\alpha \beta} & 0_{\alpha \beta}
\end{array}\right\|, & \mathfrak{B}=\left\|\begin{array}{lll}
B_{i j} & 0_{i \beta} & 0_{i \beta} \\
0_{\alpha j} & 0_{\alpha \beta} & 0_{\alpha \beta} \\
0_{\alpha j} & 0_{\alpha \beta} & 0_{\alpha \beta}
\end{array}\right\|, \\
\mathfrak{M}=\left\|\begin{array}{lll}
M_{i j} & 0_{i \beta} & 0_{i \beta} \\
0_{\alpha j} & 0_{\alpha \beta} & \delta_{\alpha \beta} \\
0_{\alpha j} & 0_{\alpha \beta} & 0_{\alpha \beta}
\end{array}\right\|, & \mathfrak{N}=\left\|\begin{array}{lll}
N_{i j} & 0_{i \beta} & 0_{i \beta} \\
0_{\alpha j} & 0_{\alpha \beta} & 0_{\alpha \beta} \\
0_{\alpha j} & 0_{\alpha \beta} & \delta_{\alpha \beta}
\end{array}\right\| .
\end{aligned}
$$

This system is seen to be self-adjoint with the matrix

$$
\mathfrak{T}=\left\|\begin{array}{ccc}
T_{i j} & 0_{i \beta} & 0_{i \beta} \\
0_{\alpha j} & 0_{\alpha \beta} & -\delta_{\alpha \beta} \\
0_{\alpha j} & \delta_{\alpha \beta} & 0_{\alpha \beta}
\end{array}\right\|,
$$

where $T \equiv\left\|T_{i j}\right\|$ is the matrix with which (2.1) is $H$-definitely self-adjoint. Condition (ii) of $\S 2$ is seen to be satisfied by this system. Since $\mathcal{L}\left[y_{\alpha}\right]=\lambda_{\alpha} B y_{\alpha}$, $s\left[y_{\alpha}\right]=0,(\alpha=1, \cdots, s-1)$, it also follows readily that if $(y, u, v)$ is a characteristic solution of (9.5), then $u_{\alpha}=0,(\alpha=1, \cdots, s-1)$; moreover, for such a characteristic solution $y \neq 0$ on $a b$. In particular, for $\lambda=0$ this result implies that whenever condition (iv) is satisfied by (2.1) this condition also holds for (9.5). Finally, if $(y, u, v)$ belongs to the corresponding linear vector space $\mathfrak{Z}$ for (9.5), then $y$ belongs to the space $L$ for (2.1); also, for such $(y, u, v)$ the corresponding functional $H[y, u, v]$ reduces simply to $H[y]$. Therefore, con- 
dition (iii)' for (2.1) implies the corresponding condition for (9.5), and if (2.1) is $H$-definitely self-adjoint so also is the latter system.

Now if $f$ belongs to the class $C_{s}$ for (2.1), the set $\left\{f_{i}, u_{\alpha} \equiv\right.$ constant, $\left.v_{\alpha}=\int_{a}^{x} y_{\alpha}(t) S(t) f(t) d t\right\}$ belongs to the corresponding class $\mathcal{V}_{1}$ for (9.5); conversely, if $\left(f_{i}, u_{\alpha}, v_{\alpha}\right)$ belongs to $\mathfrak{C}_{1}$ the vector $f$ belongs to $\boldsymbol{C}_{s}$. In particular, $\boldsymbol{C}_{\boldsymbol{s}}$ is vacuous if and only if $\mathfrak{S}_{1}$ is vacuous. If $\mathfrak{G}_{1}$ is nonvacuous, then by Theorem 9.1 the minimum of $H[y, u, v] \equiv H[y]$ in this class exists and is the smallest positive characteristic value of (9.5). Since, as pointed out above, for a characteristic solution of $(9.5)$ we have $u \equiv 0, y \neq 0$, it follows that the smallest positive characteristic value of (9.5) is a characteristic value for (2.1). It is obvious that the characteristic value thus determined is equal to $\lambda_{8}$ according to the previously introduced notation.

We are now in a position to derive a result which is complementary to that of Theorem 6.1.

THEOREM 9.3. A necessary and sufficient condition that an H-definitely selfadjoint system have at least $k$ positive [negative] characteristic values, where it is to be understood that each such value is counted a number of times equal to its multiplicity, is that the quadratic functional $\int_{a}^{b} f S f d x$ be positive [negative] definite on a linear subspace of $L$ of dimension $k$.

For suppose that positive characteristic values $\lambda_{1}, \cdots, \lambda_{k}$ exist for such a system (2.1), and that $y_{1}, \cdots, y_{k}$ are corresponding orthonormal characteristic solutions. Then for arbitrary constants $\left(d_{1}, \cdots, d_{k}\right) \neq(0, \cdots, 0)$ we have for $f=y_{1} d_{1}+\cdots+y_{k} d_{k}$ that $\int_{a}^{b} f S f d x=d_{1}^{2}+\cdots+d_{k}^{2}>0$. On the other hand, if $\int_{a}^{b} f S f d x$ is positive definite on a linear subspace of $L$ of dimension $k$, then the classes $\boldsymbol{C}_{1}, \cdots, \boldsymbol{C}_{k}$ as defined above are seen to be nonvacuous and (2.1) has at least $k$ positive characteristic values. Again, in view of the possibility of replacing $B$ by $-B$, the result for negative characteristic values is a ready consequence of the result for positive characteristic values.

We shall now give a particular condition which is sufficient to insure that an $H$-definitely self-adjoint system (2.1) has infinitely many characteristic values of a given sign. We shall denote by $\left(v_{+}\right)$the following hypothesis.

$\left(\mathrm{v}_{+}\right)$There is a subinterval $a_{1} b_{1}, a<a_{1}<b_{1}<b$, of $a b$ such that if $a_{1}^{\prime}, b_{1}^{\prime}$ are arbitrary values satisfying $a_{1} \leqq a_{1}^{\prime}<b_{1}^{\prime} \leqq b_{1}$, then there exists a vector $g(x)$ and associated $f(x)$ satisfying $\mathcal{L}[f]=B g$ for which $f \equiv 0$ outside $a_{1}^{\prime} b_{1}^{\prime}$, whereas $\int_{a}^{b} f S f d x>0$.

The condition obtained by replacing in $\left(\mathrm{v}_{+}\right)$the relation " $\int_{a}^{b} f S f d x>0$ " by " $\int_{a}^{b} f S f d x<0$ " will be referred to as ( $\left.\mathrm{v}_{-}\right)$. Using the device of the proof of Theorem 6.2, and the result of the above theorem, one obtains the following conclusion.

THEOREM 9.4. If an H-definitely self-adjoint system (2.1) satisfies hypothesis $\left(\mathrm{v}_{+}\right)$, then this system admits infinitely many positive characteristic values. Simi- 
larly, if such a system satisfies condition $\left(\mathrm{v}_{-}\right)$, there exist infinitely many negative characteristic values.

In agreement with our modified notation for the characteristic values and solutions of an $H$-definitely self-adjoint system (2.1), we write

$$
c_{\mu}[f]=\frac{\left|\lambda_{\mu}\right|}{\lambda_{\mu}} \int_{a}^{b} f S y_{\mu} d x, \quad \mu=1,-1,2,-2, \cdots .
$$

THEOREM 9.5. For an arbitrary vector $f$ of $L$,

$$
\int_{a}^{b} f S f d x=\sum_{\mu=-\infty}^{\infty} \frac{\left|\lambda_{\mu}\right|}{\lambda_{\mu}} c_{\mu}^{2}[f]
$$

moreover, if $f$ and $h$ are vectors of $L$,

$$
\int_{a}^{b} f S h d x=\sum_{\mu=-\infty}^{\infty} \frac{\left|\lambda_{\mu}\right|}{\lambda_{\mu}} \mathbf{c}_{\mu}[f] \mathbf{c}_{\mu}[h] .
$$

In view of Theorem 9.2, relation (9.6) is readily seen to be true if (2.1) admits only a finite number of characteristic values. We shall prove this relation for the case in which this system has infinitely many positive, and also infinitely many negative, characteristic values; the modification in the proof whenever the system has only a finite number of characteristic values of one sign is obvious.

Corresponding to a vector $f$ of $L$ and a given positive integer $k$, set $f_{k}=f-\sum_{\mu-k}^{k} y_{\mu}(x) c_{\mu}[f]$. Then $c_{\mu}\left[f_{k}\right]=0,(\mu=-k, \cdots, k)$, and by the minimizing properties of the characteristic values of (2.1) we have

$$
H\left[f_{k}\right] \geqq \lambda_{k+1} \int_{a}^{b} f_{k} S f_{k} d x \geqq 0
$$

if $\int_{a}^{b} f_{k} S f_{k} d x \geqq 0$; whereas

$$
H\left[f_{k}\right] \geqq \lambda_{-k-1} \int_{a}^{b} f_{k} S f_{k} d x \geqq 0
$$

if $\int_{a}^{b} f_{k} S f_{k} d x \leqq 0$. Consequently,

$$
\left|\int_{a}^{b} f_{k} S f_{k} d x\right| \leqq \max \left\{\frac{H\left[f_{k}\right]}{\lambda_{k+1}},-\frac{H\left[f_{k}\right]}{\lambda_{-k-1}}\right\} .
$$

Moreover, since

$$
0 \leqq H\left[f_{k}\right]=H[f]-\sum_{\mu=-k}^{k}\left|\lambda_{\mu}\right| c_{\mu}^{2}[f] \leqq H[f]
$$

it follows that 


$$
0=\lim _{k \rightarrow \infty} \int_{a}^{b} f_{k} S f_{k} d x=\int_{a}^{b} f S f d x-\lim _{k \rightarrow \infty} \sum_{\mu=-k}^{k} \frac{\left|\lambda_{\mu}\right|}{\lambda_{\mu}} \mathbf{c}_{\mu}^{2}[f] .
$$

Since the series involved in (9.6) clearly converges absolutely, this relation is established. Relation (9.7) is then immediate since if $f$ and $h$ are vectors of $L$, so also are $f+h$ and $f-h$, and

$$
\begin{aligned}
\int_{a}^{b} f S h d x & =\frac{1}{4}\left[\int_{a}^{b}(f+h) S(f+h) d x-\int_{a}^{b}(f-h) S(f-h) d x\right] \\
\mathbf{c}_{\mu}[f \pm h] & =\mathrm{c}_{\mu}[f] \pm \mathrm{c}_{\mu}[h] .
\end{aligned}
$$

COROLlaRY 1. If $f$ is a vector of $L^{2}$ then the equality sign holds in (6.2), that is

$$
H[f]=\sum_{\mu=-\infty}^{\infty}\left|\lambda_{\mu}\right| \mathbf{c}_{\mu}^{2}[f]
$$

Let $h$ be a vector of $L$ such that $\mathcal{L}[f]=B h, s[f]=0$. Since $\lambda_{\mu} \mathbf{c}_{\mu}[f]=\mathbf{c}_{\mu}[h]$, $(\mu=1,-1,2,-2, \cdots)$, this corollary is a consequence of $(9.7)$ and the relation $H[f]=\int_{a}^{b} f S h d x$.

It is to be noted that in general we do not have relation (9.8) for arbitrary vectors $f$ of the space $L$. This fact is shown by the example (5.11), in view of the comment immediately preceding Theorem 8.3. We do have, however, the following result.

Corollary 2. If the class $C_{s},(s=1,-1,2,-2, \cdots)$, is nonvacuous for an $H$-definitely self-adjoint system (2.1), then the minimum of $H[f]$ in this class is attained by a particular $f$ of $C_{s}$ if and only if $f=Y_{s}(x)+\Phi_{s}(x)$, where $Y_{s}(x)$ is a characteristic solution for $\lambda=\lambda_{s}$ and $\Phi_{s}$ is a vector of $L$ satisfying $B \Phi_{s} \equiv 0$ on $a b$.

By the use of inequality (6.2), relation (9.6), and an argument similar to that employed in the proof of Theorem 7.2, we have that if $f$ is a vector of a nonvacuous class $\boldsymbol{C}_{\boldsymbol{s}}$ which renders $H[f]$ its minimum value in this class, then $f=Y_{s}+\Phi_{s}$, where $Y_{s}$ is a characteristic solution of $(2.1)$ for $\lambda=\lambda_{s}$ and $\Phi_{s}$ is a vector of $L$ such that $c_{\mu}\left[\Phi_{s}\right]=0,(\mu=1,-1,2,-2, \cdots)$. It then follows from Corollary 1 to Theorem 5.4 that $\int_{a}^{b} \Phi_{s} S y d x=0$ for every vector $y$ of $L$ and thus, in particular, $\int_{a}^{b} \Phi_{s} S \Phi_{s} d x=0$. Then

$$
\begin{aligned}
0=H\left[f ; \lambda_{8}\right] & =H\left[Y_{8}: \lambda_{8}\right]+2 H\left[\Phi_{8} ; Y_{8}: \lambda_{8}\right]+H\left[\Phi_{8}: \lambda_{8}\right] \\
& =H\left[\Phi_{8}: \lambda_{8}\right]=H\left[\Phi_{8}\right],
\end{aligned}
$$

and hence $B(x) \Phi_{s}(x) \equiv 0$ on $a b$ by condition (iii)'.

THEOREM 9.6. If (2.1) is H-definitely self-adjoint and we write $v_{\mu}(x)$ $=S(x) y_{\mu}(x),(\mu=1,-1,2,-2, \cdots)$, then each of the series 


$$
\sum_{\mu=-\infty}^{\infty} \frac{\left[v_{i \mu}(x)\right]^{2}}{\left|\lambda_{\mu}\right|},
$$$$
i=1,2, \cdots, n \text {, }
$$

converges and its sum does not exceed $K_{1 i i}(x, x)$ on ab. For fixed values of one of the variables $x, t$, each of the series

$$
\sum_{\mu=-\infty}^{\infty} \frac{v_{i \mu}(x) v_{j \mu}(t)}{\left|\lambda_{\mu}\right|}, \quad i, j=1,2, \cdots, n,
$$

converges absolutely and uniformly in the other variable on ab. Finally, for an arbitrary vector $f$ of $L$ the vector series

$$
\sum_{\mu=-\infty}^{\infty} \boldsymbol{v}_{\mu}(x) \mathbf{c}_{\mu}[f]
$$

converges absolutely and uniformly on ab.

Corresponding to an arbitrary vector $g(x)$, define $f$ by $\mathcal{L}[f]=B g, s[f]=0$. Then for an arbitrary integer $k$,

$$
\begin{aligned}
0 & \leqq H\left[f-\sum_{|\mu| \leqq k} \boldsymbol{y}_{\mu} \mathbf{c}_{\mu}[f]\right] \\
& \equiv \int_{a}^{b} \int_{a}^{b} g_{i}(x)\left\{K_{1 i j}(x, t)-\sum_{|\mu| \leqq k} \frac{\boldsymbol{v}_{i \mu}(x) \boldsymbol{v}_{j \mu}(t)}{\left|\lambda_{\mu}\right|}\right\} g_{j}(t) d x d t .
\end{aligned}
$$

Applying the argument of Lemma 4.2 to the double integral of (9.12), it follows in particular that

$$
\sum_{|\mu| \leqq k} \frac{\left[v_{i \mu}(x)\right]^{2}}{\left|\lambda_{\mu}\right|} \leqq K_{1 i i}(x, x)
$$

for each integer $k$. Hence the series (9.9) converges and its sum does not exceed $K_{1 i i}(x, x)$. Since the sum of this series is uniformly bounded on $a b$, Cauchy's inequality insures that each of the series (9.10) converges absolutely and uniformly in each of the variables on $a b$ for fixed values of the other variable. In particular, each of these series defines a function which is continuous in each of the variables $x, t$ separately on $a b$. Since for an arbitrary $f$ of $L$ the series $\sum_{\mu}\left|\lambda_{\mu}\right| c_{\mu}^{2}[f]$ converges, the absolute and uniform convergence of (9.11) is a consequence of the uniform boundedness of the sum of the series (9.9) on $a b$ and Cauchy's inequality.

The proof of the above convergence properties of (9.9), (9.10) parallels that of corresponding results (see, for example, [3, p. 456]) used in establishing Theorem 8.4 for the boundary problem (8.1). We are unable to prove for (2.1) a result as general in character as Theorem 8.4 gives for system (8.1), however, since for an $H$-definite self-adjoint system we do not in general 
have that relation (9.8) is valid for arbitrary vectors $f$ of $L$. When this latter condition is fulfilled for a particular $H$-definite self-adjoint problem it then follows that the sum of the series $(9.10)$ is $K_{1 i j}(x, t)$; in particular, the sum of (9.9) is $K_{1 i i}(x, x)$, by Dini's theorem the convergence of this latter series is uniform on $a b$, and it then follows that the series (9.10) converges absolutely and uniformly in $(x, t)$ jointly.

THEOREM 9.7. For a vector $f$ of $L$ relation (9.8) holds if and only if

$$
S(x) f(x)=\sum_{\mu=-\infty}^{\infty} S(x) y_{\mu}(x) \mathbf{c}_{\mu}[f] \equiv \sum_{\mu=-\infty}^{\infty} \boldsymbol{v}_{\mu}(x) \mathbf{c}_{\mu}[f] .
$$

From the above theorem we know that the right-hand member of (9.13) converges absolutely and uniformly on $a b$, and hence defines a vector whose components are continuous on this interval. If $\mathcal{L}[f]=B g, s[f]=0$, and relation (9.13) holds for $f$, then

$$
H[f]=\int_{a}^{b} g S f d x=\sum_{\mu=-\infty}^{\infty} \mathrm{c}_{\mu}[f] \int_{a}^{b} g S y_{\mu} d x=\sum_{\mu=-\infty}^{\infty}\left|\lambda_{\mu}\right| \mathbf{c}_{\mu}^{2}[f],
$$

since $\mathbf{c}_{\mu}[g]=\lambda_{\mu} \mathbf{c}_{\mu}[f]$. On the other hand, if we define $f_{k}(x)=f(x)$ $-\sum_{|\mu| \leqq k} y_{\mu}(x) c_{\mu}[f]$, relation (9.8) is equivalent to the condition $\lim _{k \rightarrow \infty} H\left[f_{k}\right]$ $=0$, whereas (9.13) is equivalent to $F(x) \equiv \lim _{k \rightarrow \infty} S(x) f_{k}(x) \equiv 0$. In view of relation (8.13) for $f_{k}$ it then follows that if (9.8) holds for a vector $f$ then the associated vector $F$ is identically zero, that is, relation (9.13) is also valid.

Since the matrix $T$ is nonsingular, it is clear that relation (9.13) holds for a particular $f$ if and only if the series $\sum_{\mu} B(x) y_{\mu}(x) c_{\mu}[f]$ converges absolutely and uniformly on $a b$, and

$$
B(x) f(x)=\sum_{\mu=-\infty}^{\infty} B(x) y_{\mu}(x) c_{\mu}[f] .
$$

COROLlaRY. If $f$ is a vector of $L^{2}$, then the series $\phi(x)=\sum_{\mu} y_{\mu}(x) c_{\mu}[f]$ converges absolutely and uniformly on $a b$, and $B(x)[f(x)-\phi(x)] \equiv 0$.

Corollary 1 to Theorem 9.5 and Theorem 9.7 implies that relation (9.13), and hence (9.14), holds for such an $f$. We have, therefore, only to prove the absolute and uniform convergence of the series $\phi$. Let $h(x)$ be a vector of $L$ such that $\mathcal{L}[f]=B h, s[f]=0$. Now since $h$ belongs to $L$, the series $\sum_{\mu} B(x) y_{\mu}(x) c_{\mu}[h] \equiv \sum_{\mu} B(x) y_{\mu}(x) \lambda_{\mu} c_{\mu}[f]$ converges absolutely and uniformly on $a b$. Hence the corresponding convergence of $\phi$ is a consequence of

$$
\begin{aligned}
\phi(x) & =\sum_{\mu=-\infty}^{\infty}\left\{\int_{a}^{b} G(x, t) B(t) \mathbf{y}_{\mu}(t) d t\right\} \lambda_{\mu} \mathbf{c}_{\mu}[f] \\
& =\int_{a}^{b} G(x, t)\left\{\sum_{\mu=-\infty}^{\infty} B(t) y_{\mu}(t) \mathbf{c}_{\mu}[h]\right\} d t .
\end{aligned}
$$


In conclusion, we shall prove the following general expansion theorem.

THEOREM 9.8. If for an arbitrary vector $f$ the series $\sum_{\mu} B(x) y_{\mu}(x) c_{\mu}[f]$ converges uniformly and satisfies relation (9.14) on ab, then for $f^{*}(x)$ defined by the system $\mathcal{L}\left[f^{*}\right]=B f, s\left[f^{*}\right]=0$, the series $\sum_{\mu} y_{\mu}(x) c_{\mu}\left[f^{*}\right]$ converges uniformly on this interval to $f^{*}(x)$.

This result is an immediate consequence of the relations

$$
\begin{aligned}
f^{*}(x) & =\int_{a}^{b} G(x, t) B(t) f(t) d t \\
& =\sum_{\mu=-\infty}^{\infty}\left\{\int_{a}^{b} G(x, t) B(t) y_{\mu}(t) d t\right\} \mathbf{c}_{\mu}[f] \\
& =\sum_{\mu=-\infty}^{\infty} \frac{y_{\mu}(x)}{\lambda_{\mu}} c_{\mu}[f]=\sum_{\mu=-\infty}^{\infty} y_{\mu}(x) \mathbf{c}_{\mu}\left[f^{*}\right] .
\end{aligned}
$$

10. A boundary problem of the calculus of variations. We shall now consider a system of the form (2.1) associated with the problem of Bolza in the calculus of variations. The symbols $\eta \equiv\left(\eta_{i}\right), \eta^{\prime} \equiv\left(\eta_{i}^{\prime}\right)$ will denote the functions $\left[\eta_{1}(x), \cdots, \eta_{n}(x)\right]$ and the set of their derivatives, respectively. Let

$$
J[\eta]=2 Q[\eta(a), \eta(b)]+\int_{a}^{b} 2 \omega\left(x, \eta, \eta^{\prime}\right) d x,
$$

where $\omega$ and $Q$ are quadratic forms in the $2 n$ variables $\eta_{i}, \eta_{i}^{\prime}$ and $\eta_{i}(a), \eta_{i}(b)$, respectively. The functional $J[\eta]$ is of the form of the second variation of a problem of Bolza. The boundary value problem to be considered consists of the Euler-Lagrange differential equations and transversality conditions for the problem of minimizing $J[\eta]$ in a class of arcs $\eta=\eta(x)$ which satisfy a set of ordinary linear differential equations of the first order

$$
\Phi_{\alpha}\left(x, \eta, \eta^{\prime}\right) \equiv \Phi_{\alpha \pi_{i}}(x) \eta_{i}^{\prime}+\Phi_{\alpha \eta_{i}}(x) \eta_{i}=0, \quad \alpha=1, \cdots, m<n,
$$

the linear homogeneous end conditions

$$
\Psi_{\gamma}[\eta(a), \eta(b)] \equiv \Psi_{\gamma ; j a} \eta_{j}(a)+\Psi_{\gamma ; j b} \eta_{j}(b)=0, \quad \gamma=1, \cdots, p \leqq 2 n,
$$

and which render a fixed constant value to the integral

$$
\int_{a}^{b} \eta_{i} \Omega_{i j}(x) \eta_{j} d x \text {. }
$$

For the general problem of Bolza the second variation may be written as (10.1) if one includes in the set $\eta$ not only the variations of the dependent functions in the original problem of Bolza, but also two additional functions representing the variations of the end values; these latter two functions are further restricted by including in (10.2) two additional differential equations which require them to be constant on $a b$. 
Throughout the present section the following subscripts have the ranges indicated $: i, j, k=1, \cdots, n ; \alpha, \beta=1, \cdots, m ; \sigma, \tau=1, \cdots, 2 n ; \gamma, \nu=1, \cdots, p$; $\theta, \phi=1, \cdots, 2 n-p$. Partial derivatives of $\omega(x, \eta, \pi), \Phi_{\alpha}(x, \eta, \pi)$ with respect to the variables $\eta_{i}, \pi_{i}$ will be denoted by writing these variables as subscripts; correspondingly, derivatives of $Q$ and $\Psi_{\gamma}$ with respect to the arguments $\eta_{i}(a)$, $\eta_{i}(b)$ will be denoted by $Q_{i a}, \Psi_{\gamma ; i a}, Q_{i b}, \Psi_{\gamma ; i b}$, respectively.

The analysis of this section is based on the following hypotheses.

$\left(\mathrm{H}_{1}\right)$ The coefficients of the quadratic form $\omega(x, \eta, \pi)$ and the linear forms $\Phi_{\alpha}(x, \eta, \pi)$ are real single-valued functions of $x$ on $a b$. The functions $\omega_{\pi_{i} \pi_{j}}, \omega_{\pi_{i} \eta_{j}}, \Phi_{\alpha \pi_{j}}$ are of class $C^{1}$, while the functions $\pi_{\eta_{i} \eta_{j}}, \Phi_{\alpha \eta_{j}}, \Omega_{i j}=\Omega_{j i}$ are continuous on this interval. Finally, the matrix $\left\|\Phi_{\alpha \pi_{j}}(x)\right\|$ is of rank $m$ on $a b$, the coefficients of the quadratic form $Q$ and the linear forms $\Psi_{\gamma}$ are real constants, and the matrix $\left\|\Psi_{\gamma ; j a} \Psi_{\gamma ; j b}\right\|$ has rank $p$.

$\left(\mathrm{H}_{2}\right)$ The matrix

$$
\left\|\begin{array}{cc}
\omega_{\pi_{i} \pi_{j}} & \Phi_{\beta \pi_{i}} \\
\Phi_{\alpha \pi_{j}} & 0_{\alpha \beta}
\end{array}\right\|
$$

is nonsingular on $a b$.

An arc $\eta$ will be termed differentially admissible if its components $\eta_{i}(x)$ are of class $D^{1}$ on $a b$, and satisfy $\Phi_{\alpha}=0$ on this interval. An arc whose end values at $a$ and $b$ satisfy $\Psi_{\gamma}=0$ will be called terminally admissible; finally, an arc which is both differentially and terminally admissible will be said to be admissible.

$\left(\mathrm{H}_{3}\right)$ There exist $p$ differentially admissible arcs $\eta_{i}=\eta_{i v}$, such that the determinant $\left|\Psi_{\gamma}\left[\eta_{\nu}(a), \eta_{\nu}(b)\right]\right|$ is different from zero.

For arbitrary constants $\mu_{\alpha}$ define

$$
\Omega(x, \eta, \pi, \mu)=\omega(x, \eta, \pi)+\mu_{\alpha} \Phi_{\alpha}(x, \eta, \pi) .
$$

Under the hypotheses $\left(\mathrm{H}_{1}\right),\left(\mathrm{H}_{2}\right),\left(\mathrm{H}_{3}\right)$ it follows from the theory of the problem of Bolza that if $\eta(x)$ is a minimizing arc for the above defined calculus of variations problem, then there exist multipliers $\lambda=$ constant, $\mu_{\alpha}=\mu_{\alpha}(x)$ such that the set $\left[\eta_{i}(x), \mu_{\alpha}(x), \lambda\right]$ satisfies the differential equations

$$
\begin{aligned}
(d / d x) \Omega_{\pi_{i}}\left(x, \eta, \eta^{\prime}, \mu\right)-\Omega_{\eta_{i}}\left(x, \eta, \eta^{\prime}, \mu\right)+\lambda \Omega_{i j}(x) \eta_{j} & =0, \\
\Phi_{\alpha}\left(x, \eta, \eta^{\prime}\right) & =0 ;
\end{aligned}
$$

moreover, there exist constants $d_{\gamma}$ satisfying

$$
\begin{aligned}
Q_{i a}[\eta]+d_{\gamma} \Psi_{\gamma ; i a}-\left.\Omega_{\pi_{i}}\left(x, \eta, \eta^{\prime}, \mu\right)\right|^{x=a} & =0, \\
Q_{i b}[\eta]+d_{\gamma} \Psi_{\gamma ; i b}+\left.\Omega_{\pi_{i}}\left(x, \eta, \eta^{\prime}, \mu\right)\right|^{x=b} & =0, \\
\Psi_{\gamma}[\eta(a), \eta(b)] & =0 .
\end{aligned}
$$

As (10.5) is nonsingular, the set of $m+n$ equations

(10.9) $\zeta_{i}=\Omega_{\pi_{i}}(x, \eta, \pi, \mu), \quad \Phi_{\alpha}(x, \eta, \pi)=0, \quad \alpha=1, \cdots . m: i=1, \cdots, n$, 
has unique solutions

$$
\pi_{i}=\mathfrak{A}_{i j}(x) \eta_{j}+\mathfrak{B}_{i j}(x) \zeta_{j}, \quad \mu_{\alpha}=l_{\alpha j}(x) \eta_{j}+m_{\alpha j}(x) \zeta_{j} .
$$

Substituting these values in $\Omega_{\eta_{i}}(x, \eta, \pi, \mu)$, we obtain

$$
\Omega_{\eta_{i}}(x, \eta, \pi, \mu)=\mathfrak{E}_{i j}(x) \eta_{j}-\mathfrak{A}_{j i}(x) \zeta_{j},
$$

where in view of the above hypotheses the functions $\mathfrak{A}_{i j}, \mathfrak{B}_{i j}, \mathfrak{E}_{i j}$ are continuous on $a b$; moreover, the matrices $\left\|\mathfrak{B}_{i j}\right\|$ and $\left\|\mathfrak{G}_{i j}\right\|$ are symmetric and $\left\|\mathfrak{B}_{i j}\right\|$ is of rank $n-m$ on this interval. Consequently, the differential equations (10:7) are equivalent to the system

$$
\begin{aligned}
\mathcal{L}_{i}[\eta, \zeta] & \equiv \eta_{i}^{\prime}-\mathfrak{A}_{i j}(x) \eta_{j}-\mathfrak{B}_{i j}(x) \zeta_{j}=0, \\
\mathcal{L}_{n+i}[\eta, \zeta] & \equiv \zeta_{i}^{\prime}-\mathfrak{S}_{i j}(x) \eta_{j}+\mathfrak{A}_{j i}(x) \zeta_{j}=-\lambda \Re_{i j}(x) \eta_{j} .
\end{aligned}
$$

Now if $c_{i}=c_{i \theta}, d_{i}=d_{i \theta},(\theta=1, \cdots, 2 n-p)$, are linearly independent solutions of the equations $\Psi_{\gamma ; j a} c_{j}+\Psi_{\gamma ; j b} d_{j}=0,(\gamma=1, \cdots, p)$, the boundary conditions (10.8) are equivalent to the linearly independent set

$$
\begin{aligned}
s_{\gamma}[\eta, \zeta] & \equiv \Psi_{\gamma}[\eta(a), \eta(b)]=0, \\
s_{p+\theta}[\eta, \zeta] & \equiv c_{i \theta}\left\{Q_{i a}[\eta]-\zeta_{i}(a)\right\}+d_{i \theta}\left\{Q_{i b}[\eta]+\zeta_{i}(b)\right\}=0 .
\end{aligned}
$$

The system $\left(10.7^{\prime}\right),\left(10.8^{\prime}\right)$, which is clearly of the form $(2.1)$ in $y \equiv\left(\eta_{i}, \zeta_{i}\right)$, may be shown under the above hypotheses to satisfy conditions (i), (ii) and (iv) of $\$ 2$ with the matrix

$$
T \equiv\left\|\begin{array}{rr}
0_{i j} & \delta_{i j} \\
-\delta_{i j} & 0_{i j}
\end{array}\right\|
$$

For this system we have

$$
A \equiv\left\|\begin{array}{cc}
\mathfrak{A}_{i j} & \mathfrak{B}_{i j} \\
\mathfrak{S}_{i j} & -\mathfrak{A}_{j i}
\end{array}\right\|, \quad B \equiv\left\|\begin{array}{rr}
0_{i j} & 0_{i j} \\
-\mathfrak{R}_{i j} & 0_{i j}
\end{array}\right\|, \quad S \equiv\left\|\begin{array}{ll}
\mathfrak{R}_{i j} & 0_{i j} \\
0_{i j} & 0_{i j}
\end{array}\right\| .
$$

We now wish to consider the condition (iii)' for such a system. The linear vector space $L$ for this problem consists of sets $(\eta, \zeta)$ which satisfy with a corresponding $w \equiv\left(w_{i}\right)$ the system

$$
\eta_{i}^{\prime}=\mathfrak{A}_{i j} \eta_{j}+\mathfrak{B}_{i j} \zeta_{j}, \quad \zeta_{i}^{\prime}=\mathfrak{S}_{i j} \eta_{j}-\mathfrak{A}_{j i} \zeta_{j}-\mathfrak{R}_{i j} w_{j}, \quad s_{\sigma}[\eta, \zeta]=0 .
$$

It is readily seen from $(10.8)$ that if $s_{\sigma}[\eta, \zeta]=0$, then

$$
\left.\eta_{i} \zeta_{i}\right|_{c} ^{b}=-2 Q[\eta]
$$

Evaluating $H=H[\eta, \zeta]$ for such a set $(\eta, \zeta)$ we find

$$
H=-\left.\eta_{i} \zeta_{i}\right|_{a} ^{b}+\int_{a}^{b}\left(\eta_{i}^{\prime} \zeta_{i}+\eta_{i} \mathcal{S}_{i j} \eta_{j}-\eta_{i} \mathfrak{A}{ }_{j i} \zeta_{j}\right) d x=J[\eta],
$$


in view of (10.9) and (10.11). Consequently, (iii)' for this system reduces to the condition that $J[\eta]>0$ for every set $(\eta, \zeta)$ which satisfies with an associated vector w the system (10.12), and for which $\left(\Omega_{i j} \eta_{j}\right) \not \equiv\left(0_{i}\right)$ on $a b$. If $(\eta, \zeta)$ is a solution of (10.12) for a given vector $w$, then clearly $\eta$ is an admissible arc. Hence (iii)' is certainly satisfied if the following condition holds.

$\left(\mathrm{H}_{4}\right) J[\eta]>0$ for arbitrary nonidentically vanishing admissible arcs $\eta$.

We thus see that a system of the form treated by Reid [9] for which, using the notation of that paper, the quadratic form $G[\eta(a), \eta(b)]$ is identically zero, is $H$-definitely self-adjoint.

THEOREM 10.1. Suppose that a problem of the above sort is H-definitely selfadjoint. If the matrix $\AA(x)$ is positive [negative] definite at a point $x_{0}$ of $a b$, then this system has infinitely many positive [negative] characteristic values.

On the assumption that $\Re\left(x_{0}\right)$ is positive definite, there clearly exists a subinterval $a_{1} b_{1}, a<a_{1}<b_{1}<b$, throughout which $\Omega(x)$ remains positive definite. Corresponding to an arbitrary subinterval $a_{1}^{\prime} b_{1}^{\prime}$ of $a_{1} b_{1}$, denote by $\zeta \equiv\left(\zeta_{i_{\rho}}\right),(\rho=1, \cdots, n+1)$, a set of $n+1$ vectors whose components are of class $C^{1}$ on $a b$, are identically zero outside the subinterval $a_{1}^{\prime} b_{1}^{\prime}$, and such that the vectors $\left(\mathfrak{B}_{i j} \zeta_{j \rho}\right),(\rho=1, \cdots, n+1)$, are linearly independent on $a_{1}^{\prime} b_{1}^{\prime}$. Such vectors clearly exist since $\mathfrak{B}$ is of rank $n-m$ on $a b$; in fact, all that is necessary to insure the existence of such vectors is that $\mathfrak{B} \not \equiv 0$ on $a_{1}^{\prime} b_{1}^{\prime}$. Now define $\eta_{\rho}$ as the solution of $\eta_{i \rho}^{\prime}=\mathfrak{A}_{i j}(x) \eta_{j \rho}+\mathfrak{B}_{i j}(x) \zeta_{i_{\rho}}(x), \eta_{i_{\rho}}(a)=0$. Clearly the vectors $\eta_{\rho}$ are linearly independent on $a b$; moreover, there exist constants $\left(d_{1}, \cdots, d_{n+1}\right)$ not all zero such that if we set $\eta=\eta_{1} d_{1}+\cdots+\eta_{n+1} d_{n+1}$, then $\eta_{i}(b)=0$. This vector satisfies with $\zeta=\zeta_{1} d_{1}+\cdots+\zeta_{n+1} d_{n+1}$ the system $\eta_{i}^{\prime}=\mathfrak{A}_{i j} \eta_{j}+\mathfrak{B}_{i j} \zeta_{j}$ on $a b$; furthermore, as $\zeta_{i} \equiv 0_{i}$ outside $a_{1}^{\prime} b_{1}^{\prime}$, it follows from the conditions $\eta_{i}(a)=0=\eta_{i}(b)$ that $\eta_{i} \equiv 0_{i}$ outside this subinterval. Since $\Omega$ is nonsingular on $a_{1}^{\prime} b_{1}^{\prime}$ there clearly exists a corresponding $w$ such that the differential equations of $(10.12)$ are satisfied by $(\eta, \zeta, w)$; the boundary conditions are also satisfied by $(\eta, \zeta)$ since this set vanishes at $x=a$ and $x=b$. Consequently, since on $a_{1}^{\prime} b_{1}^{\prime}$ we have that $\eta \neq 0$ and the matrix $\Omega$ is positive definite, while $\eta \equiv 0$ outside this subinterval, it follows that the thus determined solution $y=(\eta, \zeta)$ of $(10.12)$ satisfies the conditions described in hypothesis $\left(\mathrm{v}_{+}\right)$of $\$ 9$. Hence by Theorem 9.4 the considered system has infinitely many positive characteristic values. The corresponding result for negative characteristic values is readily deducible from the above by considering the related boundary value problem obtained by replacing the matrix $\Re(x)$ by $-\Omega(x)$.

11. A particular differential system. Krein [7] and Kamke [6] have considered a self-adjoint boundary problem of the form

$$
\mathcal{L}[u]=\lambda k(x) u, \quad U_{\sigma}[u]=0, \quad \sigma=1, \cdots, 2 n,
$$

where $\mathcal{L}[u]$ is a differential operator of the form 


$$
\mathcal{L}[u]=\sum_{\nu=0}^{n}\left[l_{\nu}(x) u^{(\nu)}\right]^{(\nu)},
$$

$l_{n}(x) \neq 0$ and $l_{\nu}(x),(\nu=1, \cdots, n)$, of class $C^{(\nu)}$ on $a b$, while the $U_{\sigma}[u]$ are independent linear forms in the end values of $u, u^{\prime}, \cdots, u^{(2 n-1)}$ at $x=a$ and $x=b$. Each of these authors has assumed that the functional

$$
\int_{a}^{b} u \mathcal{L}[u] d x
$$

possesses certain properties of definiteness.

Krein has supposed that (11.2) is non-negative for every function $u$ which is of class $C^{(2 n)}$ on $a b$ and such that $u, u^{\prime}, \cdots, u^{(n-1)}$ all vanish at $a$ and $b$; moreover, that the continuous function $k(x)$ occurring in (11.1) is non-negative throughout $a b$. Kamke [6, I] has assumed that (11.2) is non-negative for every function $u$ of class $C^{(2 n)}$ which satisfies the boundary conditions $U_{\sigma}[u]=0$; moreover, that $\lambda=0$ is not a characteristic value of (11.1). In addition, Kamke has also treated the case in which the continuous function $k(x)$ changes sign on $a b$.

It will now be shown that a system (11.1) may be written as one of the type considered in the preceding section. Now $\mathcal{L}[u]$ is the Euler expression for the integral

$$
\int_{a}^{b}\left\{l_{0}(x) u^{2}-l_{1}(x) u^{\prime 2}+\cdots+(-1)^{n} l_{n}(x)\left[u^{(n)}\right]^{2}\right\} d x .
$$

This integral, by a device familiar in the calculus of variations, is equivalent under the substitution $\eta_{1} \equiv u$ to the integral

$$
\int_{a}^{b}\left\{l_{0}(x) \eta_{1}^{2}-l_{1}(x) \eta_{2}^{2}+\cdots+(-1)^{n-1} \eta_{n}^{2}+(-1)^{n} \eta_{n}^{\prime 2}\right\} d x
$$

together with the auxiliary linear differential equations

$$
\Phi_{\alpha} \equiv \eta_{\alpha}^{\prime}-\eta_{\alpha+1}=0, \quad \alpha=1, \cdots, n-1 .
$$

Suppose $u$ is of class $C^{(2 n)}$ and satisfies the nonhomogeneous differential equation

$$
\mathcal{L}[u]+f(x)=0 .
$$

If we set

$$
\begin{aligned}
& \eta_{1} \equiv u, \quad \eta_{2} \equiv u^{\prime}, \cdots, \eta_{n} \equiv u^{(n-1)} \\
& \zeta_{i} \equiv(-1)^{i}\left\{\left[l_{i}(x) u^{(i)}\right]+\left[l_{i+1}(x) u^{(i+1)}\right]^{\prime}+\cdots+\left[l_{n}(x) u^{(n)}\right]^{(n-i)}\right\}, \\
& i=1, \cdots, n,
\end{aligned}
$$

it is readily seen that $\left(\eta_{i}, \zeta_{i}\right)$ satisfy the first order system 


$$
\begin{aligned}
\eta_{\alpha}^{\prime} & =\eta_{\alpha+1}, \\
\eta_{n}^{\prime} & =\frac{(-1)^{n}}{l_{n}(x)} \zeta_{n}, \\
\zeta_{1}^{\prime} & =l_{0}(x) \eta_{1}+f(x), \\
\zeta_{1+\alpha}^{\prime} & =(-1)^{\alpha} l_{\alpha}(x) \eta_{1+\alpha}-\zeta_{\alpha}, \quad \alpha=1, \cdots, n-1 .
\end{aligned}
$$

Conversely, if $\left(\eta_{i}, \zeta_{i}\right)$ is a solution of $(11.8)$ it follows that $u \equiv \eta_{1}$ satisfies (11.6); moreover, $u$ and its first $2 n-1$ derivatives are related to $\left(\eta_{i}, \zeta_{i}\right)$ by the equations (11.7). Hence there is complete equivalence between the single linear equation (11.6) of order $2 n$ and the system (11.8) of $2 n$ linear differential equations of the first order. For $f(x) \equiv 0$ this latter system is the canonical form of the Euler-Lagrange equations for the integral (11.4) subject to the auxiliary differential equations (11.5).

Now the $U_{\sigma}[u]$ are supposed to be $2 n$ independent linear forms in the end values of $u, u^{\prime}, \cdots, u^{(2 n-1)}$ at $x=a$ and $x=b$. In view of the assumption that $l_{n}(x) \neq 0$ on $a b$ it follows that they may equally well be considered as independent linear forms in the end values of the corresponding $\eta_{i}, \zeta_{i}$ at $a$ and $b$; consequently, the set $U_{\sigma}[u]=0$ may be written as

$$
\begin{aligned}
s_{\sigma}[\eta, \zeta] \equiv a_{\sigma j}^{1} \eta_{j}(a)-b_{\sigma j}^{1} \zeta_{j}(a)+a_{\sigma j}^{2} \eta_{j}(b)+b_{\sigma j}^{2} \zeta_{j}(b) & =0, \\
\sigma & =1, \cdots, 2 n .
\end{aligned}
$$

If $u$ and $u^{*}$ are of class $C^{(2 n)}$ on $a b$, it follows from the self-adjoint character of $\mathcal{L}[u]$ that (see, for example $[5$, p. 123])

$$
u^{*} \mathcal{L}[u]-u \mathcal{L}\left[u^{*}\right] \equiv \frac{d}{d x} P\left(u ; u^{*}\right)
$$

where $P\left(u ; u^{*}\right)$ is bilinear in the sets $\left(u, u^{\prime}, \cdots, u^{(2 n-1)}\right)$ and $\left(u^{*}, u^{* i}, \cdots\right.$, $\left.u^{*(2 n-1)}\right)$, and is the so-called bilinear concomitant. In particular, if $\left(\eta_{i}, \zeta_{i}\right)$ and $\left(\eta_{i}^{*}, \zeta_{i}^{*}\right)$ are defined by $(11.7)$ for $u$ and $u^{*}$, respectively, it may be readily verified that

$$
P\left(u ; u^{*}\right) \equiv \eta_{i}(x) \zeta_{i}^{*}(x)-\zeta_{i}(x) \eta_{i}^{*}(x) .
$$

The self-adjoint character of the boundary conditions implies that for arbitrary functions $u, u^{*}$ whose end values satisfy $U_{\sigma}[u]=0=U_{\sigma}\left[u^{*}\right],(\sigma=1, \cdots, 2 n)$, we have $\left.P\left(u ; u^{*}\right)\right|_{a} ^{b}=0$. Consequently, if $\left(\eta_{i}, \zeta_{i}\right)$ and $\left(\eta_{i}^{*}, \zeta_{i}^{*}\right)$ are arbitrary sets of functions satisfying $s_{\sigma}[\eta, \zeta]=0=s_{\sigma}\left[\eta^{*}, \zeta^{*}\right]$ we must have

$$
\eta_{i}(x) \zeta_{i}^{*}(x)-\left.\zeta_{i}(x) \eta_{i}^{*}(x)\right|_{a} ^{b}=0 .
$$

Now the general solution of $s_{\sigma}[\eta, \zeta]=0,(\sigma=1, \cdots, 2 n)$, is of the form 


$$
\begin{array}{ll}
\eta_{j}(a)=\xi_{\tau} c_{\tau j}^{1}, & \zeta_{j}(a)=\xi_{\tau} d_{\tau j}^{1}, \\
\eta_{j}(b)=\xi_{\tau} c_{\tau j}^{2}, & \zeta_{j}(b)=-\xi_{\tau} d_{\tau j}^{2},
\end{array}
$$

where $\left(c_{j}^{1}, d_{j}^{1}, c_{j}^{2}, d_{j}^{2}\right)=\left(c_{\tau j}^{1}, d_{\tau j}^{1}, c_{\tau j}^{2}, d_{\tau j}^{2}\right),(\tau=1, \cdots, 2 n)$, are $2 n$ linearly independent solutions of the system

$$
a_{\sigma j}^{1} c_{j}^{1}-b_{\sigma j}^{1} d_{j}^{1}+a_{\sigma j}^{2} c_{j}^{2}-b_{\sigma j}^{2} d_{j}^{2}=0, \quad \sigma=1, \cdots, 2 n .
$$

Corresponding to $\xi \equiv\left(\xi_{\sigma}\right), \xi^{*} \equiv\left(\xi_{\sigma}^{*}\right)$, determine the end values of $\left(\eta_{i}, \zeta_{i}\right)$ and $\left(\eta_{i}^{*}, \zeta_{i}^{*}\right)$ by equations (11.11). Because of the arbitrariness of $\xi$ and $\xi^{*}$ relation (11.10) then implies

$$
d_{\sigma j}^{1} c_{\tau j}^{1}-c_{\sigma j}^{1} d_{\tau j}^{1}+d_{\sigma j}^{2} c_{\tau j}^{2}-c_{c_{j}}^{2} d_{\tau j}^{2}=0, \quad \sigma, \tau=1, \cdots, 2 n,
$$

whence it follows that there is a nonsingular matrix $\left\|E_{\sigma r}\right\|$ such that

$$
d_{\sigma j}^{1}=E_{\sigma \tau} a_{\tau j}^{1}, \quad c_{\sigma j}^{1}=E_{\sigma \tau} b_{\tau j}^{1}, \quad d_{\sigma j}^{2}=E_{\sigma \tau} a_{\tau}^{2}, \quad c_{\sigma j}^{2}=E_{\sigma \tau} b_{\tau j}^{2} .
$$

Consequently, writing $\xi_{\sigma} E_{\sigma \tau}=e_{\tau}$, a set $\left(\eta_{i}, \zeta_{i}\right)$ is seen to satisfy (11.9) if and only if there are constants $\left(e_{\tau}\right)$ such that

$$
\begin{array}{ll}
\eta_{j}(a)=e_{\tau} b_{\tau j}^{1}, & \zeta_{j}(a)=e_{\tau} a_{\tau j}^{1}, \\
\eta_{j}(b)=e_{\tau} b_{\tau j}^{2}, & \zeta_{j}(b)=-e_{\tau} a_{\tau j}^{2} .
\end{array}
$$

Either from (11.12), or from substitution of $\left(11.11^{\prime}\right)$ in (11.10), it follows that the $2 n \times 2 n$ matrix

$$
\left\|k_{\sigma \tau}\right\| \equiv\left\|a_{\sigma j}^{1} b_{\tau j}^{1}+a_{\sigma j}^{2} b_{\tau j}^{2}\right\|
$$

is symmetric. Now if the $2 n \times 2 n$ matrix $\left\|b_{\sigma j}^{1} b_{\sigma j}^{2}\right\|$ is of rank $2 n-p$, denote by $r \equiv\left(r_{\sigma}\right) \equiv\left(r_{\gamma \sigma}\right),(\gamma=1, \cdots, p)$, a set of $p$ linearly independent solutions of the equations

$$
r_{\sigma} b_{\sigma j}^{1}=0, \quad r_{\sigma} b_{\sigma j}^{2}=0, \quad j=1, \cdots, n .
$$

If $\left(\eta_{i}, \zeta_{i}\right)$ satisfies $(11.9)$, then $\eta_{j}(a), \eta_{j}(b)$ must satisfy

$$
\Psi_{\gamma} \equiv \Psi_{\gamma ; j a} \eta_{j}(a)+\Psi_{\gamma ; j b} \eta_{j}(b)=0,
$$$$
\gamma=1, \cdots, p,
$$

where $\Psi_{\gamma ; j a}=r_{\gamma \sigma} a_{\sigma j}^{1}, \Psi_{\gamma ; \zeta b}=r_{\gamma \sigma} a_{\sigma j}^{2}$. Since $s_{\sigma}[\eta, \zeta]$ are independent linear forms, the $p$ conditions (11.14) are seen to be also linearly independent.

If $p=2 n$, the problem (11.1) is then seen to be equivalent to one of the sort studied in $\$ 10$ with $2 \omega$ defined as the integrand of (11.4), the auxiliary differential equations $\Phi_{\alpha}=0$ and boundary conditions determined by (11.5) and (11.14), respectively, the quadratic form $Q \equiv 0$, while the matrix $\Omega(x)$ is defined as

$$
\Re(x) \equiv\left\|\begin{array}{cc}
-k(x) & 0_{\beta} \\
0_{\alpha} & 0_{\alpha \beta}
\end{array}\right\|, \quad \alpha, \beta=1, \cdots, n-1 .
$$


In general, it is to be noted that $b_{\sigma j}^{1} \Psi_{\gamma ; j a}+b_{\sigma j}^{2} \Psi_{\gamma ; j b}=0, \quad(\sigma=1, \cdots, 2 n$; $\gamma=1, \cdots, p)$, and since the linear forms of (11.14) are independent and $\left\|b_{\sigma}^{1}, b_{\sigma j}^{2}\right\|$ is of rank $2 n-p$ it follows that if $b_{\sigma j}^{1} w_{j a}+b_{\sigma j}^{2} w_{j b}=0,(\sigma=1, \cdots, 2 n)$, then there must exist constants $d_{\gamma}$ such that $w_{j a}=d_{\gamma} \Psi_{\gamma ; j a}, w_{j b}=d_{\gamma} \Psi_{\gamma ; j b}$, $(j=1, \cdots, n)$. Moreover, for $\left(e_{\tau}\right)$ related to the end values of $\left(\eta_{i}, \zeta_{i}\right)$ by $\left(11.11^{\prime}\right)$ we have

$$
a_{\sigma j}^{1} \eta_{j}(a)+a_{\sigma j}^{2} \eta_{j}(b)=k_{\sigma \tau} e_{\tau} .
$$

Since $r \equiv\left(r_{\gamma \sigma}\right),(\gamma=1, \cdots, p)$, satisfies $k_{\sigma \tau} r_{\tau}=0$, the rank of $\left\|k_{\sigma \tau}\right\|$ does not exceed $2 n-p$. If this matrix is of rank $2 n-q$, denote by $\rho \equiv\left(\rho_{\tau}\right) \equiv\left(\rho_{v \tau}\right)$, $(\nu=1, \cdots, q)$, sets orthonormal in the sense that $\rho_{\nu \tau} \rho_{\chi \tau}=\delta_{\nu \chi},(\nu, \chi=1, \cdots, q)$, and satisfying $k_{\sigma \tau} \rho_{\tau}=0,(\sigma=1, \cdots, 2 n)$. Then the matrix

$$
\left\|\begin{array}{ll}
k_{\sigma \tau} & \rho_{X \sigma} \\
\rho_{\nu \tau} & 0_{\nu x}
\end{array}\right\|
$$

is nonsingular, and its reciprocal is a symmetric matrix of the form

$$
\left\|\begin{array}{ll}
h_{\sigma \tau} & \rho_{x \sigma} \\
\rho_{\nu \tau} & 0_{\nu x}
\end{array}\right\| .
$$

If now $\left(\eta_{i}, \zeta_{i}\right)$ satisfies (11.9), and $\left(e_{\tau}\right)$ is determined by $\left(11.11^{\prime}\right)$, it follows from (11.16) that there exist constants $t_{\nu},(\nu=1, \cdots, q)$, such that

$$
e_{\sigma}=h_{\sigma \tau}\left[a_{\tau j}^{1} \eta_{j}(a)+a_{\tau j}^{2} \eta_{j}(b)\right]+t_{\nu} \rho_{\nu \sigma}
$$

Writing

$$
2 Q[\eta] \equiv\left[a_{\sigma i}^{1} \eta_{i}(a)+\underset{a_{\sigma i} \eta_{i}}{2}(b)\right] h_{\sigma \tau}\left[a_{\tau j}^{1} \eta_{j}(a)+a_{\tau j}^{2} \eta_{j}(b)\right],
$$

it then follows from (11.11') and (11.17) that

$$
\begin{aligned}
& Q_{i a}[\eta]+t_{\nu} \rho_{\nu \tau} a_{\tau i}^{1}-\zeta_{i}(a)=0, \\
& Q_{i b}[\eta]+t_{\nu} \rho_{\nu \tau} a_{\tau i}^{2}+\zeta_{i}(b) \doteq 0, \quad i=1, \cdots, n \text {. }
\end{aligned}
$$

On the other hand, since

$$
b_{\sigma j}^{1}\left(t_{\nu} \rho_{\nu \tau} a_{\tau j}^{1}\right)+b_{\sigma j}^{2}\left(t_{\nu} \rho_{\nu \tau} a_{\tau j}^{2}\right)=t_{\nu}\left(\rho_{\nu \tau} k_{\tau \sigma}\right)=0,
$$

there exist constants $d_{\gamma}$ such that $t_{\nu} \rho_{\nu \tau} a_{\tau j}^{1}=d_{\gamma} \Psi_{\gamma ; j a}, t_{\nu} \rho_{\nu \tau} a_{\tau j}^{2}=d_{\gamma} \Psi_{\gamma ; j b}$. It thus follows that (11.1) is equivalent to a boundary value problem of the type treated in the preceding section with $2 \omega$ defined as the integrand of (11.4), the auxiliary differential equations and end conditions defined by (11.5) and (11.14), respectively, the quadratic form $Q$ of $(11.18)$, and $\Omega(x)$ given in (11.15). 
Finally, if $u$ is of class $C^{(2 n)}$ on $a b$ and $\left(\eta_{i}, \zeta_{i}\right)$ are defined by (11.7), it is readily seen that $u \mathcal{L}[u] \equiv 2 \omega\left(x, \eta, \eta^{\prime}\right)-\left(\eta_{i} \zeta_{i}\right)^{\prime}$ and

$$
\int_{a}^{b} u \mathcal{L}[u] d x \equiv \int_{a}^{b} 2 \omega\left(x, \eta, \eta^{\prime}\right) d x-\left.\eta_{i} \zeta_{i}\right|_{a} ^{b}=\int_{a}^{b} 2 \omega\left(x, \eta, \eta^{\prime}\right) d x+2 Q[\eta]
$$

whenever $U_{\sigma}[u]=0$. In particular, the hypotheses of Kamke on (11.1) are seen to imply that the above defined equivalent problem is $H$-definitely selfadjoint in the sense of $\$ 2$. Actually, a self-adjoint system (11.1) is $H$-definitely self-adjoint if $\lambda=0$ is not a characteristic value, and the functional (11.2) is non-negative for arbitrary functions $u$ of class $C^{(2 n)}$ satisfying with a continuous function $g(x)$ the system $L[u]=k(x) g(x), U_{\sigma}[u]=0$. In case $k(x)$ vanishes or changes sign on $a b$ this condition is slightly weaker than that used by Kamke.

In conclusion, it is to be remarked that once the symmetry of $\left\|k_{\sigma \tau}\right\|$ is established, the existence of linear forms $\Psi_{\gamma}$ and a quadratic form $Q$ such that the boundary conditions $s_{\sigma}[\eta, \zeta]=0$ reduce to (11.14), (11.19) has been proved by $\mathrm{Hu}[4, \mathrm{pp} .380-382]$. The above presentation, however, determines more explicitly the form of the $\Psi_{\gamma}$ and $Q$ in terms of the coefficients of the forms $s_{\sigma}[\eta, \zeta]$.

12. $H$-definitely self-conjugate adjoint systems. In the preceding sections we have been concerned with a system (2.1) involving real-valued coefficients. However, the notion of $H$-definite self-adjointness may be extended to a system (2.1) whose coefficients are complex-valued in a manner previously presented by Reid [11] for extending the notion of definite self-adjointness to such a system.

In the following we shall therefore suppose that the elements of $A(x)$, $B(x)$ are complex-valued continuous functions of the real variable $x$ on $a b$, and that the coefficient matrices $M$ and $N$ of the linearly independent boundary conditions $s_{i}[y]=0$ have complex-valued elements. If $K \equiv\left\|K_{i j}\right\|$, then we shall denote by $\bar{K}$ the matrix $\left\|\bar{K}_{i j}\right\|$ whose elements are the complex conjugates of the corresponding elements of $K$; moreover, $K^{*}$ shall denote the conjugate transpose matrix $\left\|\bar{K}_{j i}\right\|$. As in Reid [11] we shall also consider the system

$$
u^{\prime}+u \bar{A}=-\lambda u \bar{B}, \quad \bar{t}[u] \equiv u(a) \bar{P}+u(b) \bar{Q}=0,
$$

where $P$ and $Q$ are the matrices occurring in the boundary conditions of the adjoint system (2.2). System (12.1) is termed the conjugate adjoint of (2.1). The system (2.1) is said to be self-conjugate adjoint with the matrix $T$ if it is equivalent to (12.1) under the transformation $u=T(x) y$, where the elements of $T(x)$ are complex-valued functions which are of class $C^{1}$ on $a b$, and $T$ is nonsingular on this interval. It follows (Reid [11, Theorem 2.1]) that (2.1) is self-conjugate adjoint with $T(x)$ if and only if 


$$
\begin{gathered}
T A+A^{*} T+T^{\prime} \equiv 0, \quad T B+B^{*} T \equiv 0 \quad \text { on } \quad a b \\
M T^{-1}(a) M^{*} \equiv N T^{-1}(b) N^{*} .
\end{gathered}
$$

We shall now say that (2.1) is $H$-definitely self-conjugate adjoint with the matrix $T$, or merely $H$-definitely self-conjugate adjoint if :

(i) The system is self-conjugate adjoint with $T$.

(ii) The matrix $S(x) \equiv T^{*}(x) B(x)$ is hermitian.

(iii) If the linear vector space $L$ be defined as in $\$ 2$, with the understanding now that the components of $y$ and $g$ are complex-valued, then the functional

$$
H[y] \equiv \int_{a}^{b} \bar{y} T^{*} \mathcal{L}[y] d x
$$

which is readily seen to be real-valued on this space $L$, is positive for arbitrary vectors $y$ of $L$ such that $B y \not \equiv 0$ on $a b$.

(iv) There exists no nonidentically vanishing solution $y$ of $\mathcal{L}[y]=0$, $s[y]=0$ such that $B y \equiv 0$ on $a b$.

THEOREM 12.1. All the characteristic values of an H-definitely self-conjugate adjoint system (2.1) are real.

For if $y$ were a characteristic solution of an $H$-definitely self-conjugate adjoint system corresponding to a non-real characteristic value $\lambda$, it would follow as in the proof of Theorem 3.1 of Reid [11] that $\int_{a}^{b} \bar{y} S y d x=0$. Since for such a characteristic solution we have $H[y]=\lambda \int_{a}^{b} \bar{y} S y d x$, it then ensues that $H[y]=0$. Because of the above condition (iii) it would then follow that $B y \equiv 0$ on $a b$, which is impossible for a characteristic solution by condition (iv). Hence all the characteristic values of such a system are real.

Once this result is obtained, the consideration of the existence of characteristic values and related expansion theorems for an $H$-definitely self-conjugate adjoint system (2.1) is reducible to the same consideration for an associated $H$-definitely self-adjoint system with real coefficients. Since this reduction is attained by the same device of separating real and pure imaginary parts of (2.1) for real values of $\lambda$ as used in Reid [11], the details of the reduction will be left to the reader.

In a general discussion of boundary value problems one might very well start with a system of the form (2.1) whose coefficients are complex-valued, which satisfies the above conditions (i), (ii), (iv) and the following alternative to the above condition (iii):

(iii)* If the linear vector space $L$ be defined as in $\$ 2$, with the understanding that the components of $y$ and $g$ are complex-valued, then there exist real constants $\alpha$ and $\beta$ not both zero and such that the functional

$$
\int_{a}^{b} \bar{y} T^{*}(\alpha L[y]+\beta B y) d x
$$


which is readily seen to be real on $L$, is positive for arbitrary vectors $y$ of $L$ such that $B y \neq 0$ on $a b$.

If a system (2.1) satisfies (i), (ii), (iii)* and (iv), and has $\alpha \neq 0$ in (12.3), this system may be reduced to an $H$-definitely self-conjugate adjoint system by a linear change of parameter and the possibly needed change of replacing $T$ by $-T$. If for such a system we have $\alpha=0$, then the system obtained is somewhat more general than a definitely self-conjugate adjoint system; for such a problem, however, one is still able by the usual method of proof to establish the reality of characteristic values, the equality of index and multiplicity of its characteristic values, and a completeness property of the totality of characteristic solutions similar to that proved by Bliss for definitely self-adjoint systems (see Bliss [2, Theorem 2.3 and its Corollaries]). In a recent course on boundary value problems the author has followed this order of presentation. For the purpose of publication of new results, however, the above separate treatment of $H$-definitely self-adjoint systems seems desirable, since by this procedure one is able on various occasions to utilize readily certain results that have previously been established by Bliss and the author.

\section{BIBLIOGRAPHY}

1. G. A. Bliss, $A$ boundary value problem for a system of ordinary linear differential equations of the first order, these Transactions, vol. 28 (1926), pp. 561-589.

2. - Definitely self-adjoint boundary value problems, these Transactions, vol. 44 (1938), pp. 413-428.

3. E. Goursat, Cours d'Analyse Mathêmatique, vol. 3, Paris, 1927.

4. K. S. Hu, The problem of Bolza and its accessory boundary value problem, Contributions to the Calculus of Variations, 1931-1932, The University of Chicago Press, pp. 361-443.

5. E. L. Ince, Ordinary Differential Equations, 1927.

6. E. Kamke, Über die definiten selbstadjungierten Eigenwertaufgaben bei gewöhnlichen linearen Differentialgleichungen. I, Mathematische Zeitschrift, vol. 45 (1939), pp. 759-787; II, ibid., vol. 46 (1940), pp. 231-250; III, ibid., vol. 46 (1940), pp. 251-286.

7. M. Krein, Sur les opérateurs différentiels autoadjoints et leurs fonctions de Green symétriques, Recueil Mathématique, vol. 2 (44) (1937), pp. 1023-1070.

8. J. Mercer, Functions of positive and negative type, and the connection with the theory of integral equations, Philosophical Transactions of the Royal Society, vol. 209 A (1909), pp. 415446.

9. W. T. Reid, $A$ boundary value problem associated with the calculus of variations, American Journal of Mathematics, vol. 54 (1932), pp. 769-790.

10. - A system of ordinary linear differential equations with two-point boundary conditions, these Transactions, vol. 44 (1938), pp. 508-521.

11. - Some remarks on linear differential systems, Bulletin of the American Mathematical Society, vol. 45 (1939), pp. 414-419.

University of Chicago, Chicago, Ill. 